\title{
Maximal electric dipole moments of nuclei with enhanced Schiff moments
}

\author{
John Ellis, ${ }^{a, b}$ Jae Sik Lee $^{c}$ and Apostolos Pilaftsis ${ }^{d}$ \\ a Theory Division, CERN, \\ CH-1211 Geneva 23, Switzerland \\ ${ }^{b}$ Theoretical Particle Physics and Cosmology Group, Department of Physics, \\ King's College London, London WC2R 2LS, U.K. \\ ${ }^{c}$ Physics Division, National Center for Theoretical Sciences, \\ Hsinchu, 300, Taiwan \\ ${ }^{d}$ School of Physics and Astronomy, University of Manchester, \\ Manchester M13 9PL, U.K. \\ E-mail: John.Ellis@cern.ch, jslee@phys.cts.nthu.edu.tw, \\ apostolos.pilaftsis@manchester.ac.uk
}

ABstract: The electric dipole moments (EDMs) of heavy nuclei, such as ${ }^{199} \mathrm{Hg},{ }^{225} \mathrm{Ra}$ and ${ }^{211} \mathrm{Rn}$, can be enhanced by the Schiff moments induced by the presence of nearby parity-doublet states. Working within the framework of the maximally CP-violating and minimally flavour-violating (MCPMFV) version of the MSSM, we discuss the maximal values that such EDMs might attain, given the existing experimental constraints on the Thallium, neutron and Mercury EDMs. The maximal EDM values of the heavy nuclei are obtained with the help of a differential-geometrical approach proposed recently that enables the maxima of new CP-violating observables to be calculated exactly in the linear approximation. In the case of ${ }^{225} \mathrm{Ra}$, we find that its EDM may be as large as 6 to $50 \times 10^{-27} \mathrm{e} \cdot \mathrm{cm}$.

KeYwORDS: Supersymmetry Phenomenology 


\section{Contents}

1 Introduction 1

2 Schiff moments for selected nuclei 2

3 Differential-geometrical optimization method 5

$\begin{array}{lll}4 & \text { Results } & \mathbf{7}\end{array}$

4.1 The MCPMFV SUSY model with 6 CP phases $\quad 7$

$\begin{array}{lll}4.2 \text { The 7D case of non-zero } \theta_{\mathrm{QCD}} & 15\end{array}$

$\begin{array}{llr}5 & \text { Conclusions } & 18\end{array}$

\section{Introduction}

Electric dipole moments (EDMs) are among the most promising potential signatures for CP-violating physics beyond the Standard Model (SM), and one of the most promising options for extending the SM is provided by supersymmetry (SUSY) (for recent reviews, see $[1,2]$ ). The minimal SUSY extension of the SM (the MSSM) already contains many possible CP-violating phases, even in its minimally flavour-violating (MFV) version. The maximally CP-violating MFV version of the MSSM, the MCPMFV model ([3]; for related approaches, see [4-9]), has six CP-violating phases, to which may be added the QCD vacuum phase $\theta_{\mathrm{QCD}}$. These phases are tightly constrained by the present experimental upper limits on the EDMs of ${ }^{205} \mathrm{Tl}$, the neutron and ${ }^{199} \mathrm{Hg}$. Nevertheless, one could in principle envisage (accidental) cancellations $[10,11]$ between the contributions to the measured EDMs of the six (seven) phases of the MCPMFV model (including $\theta_{\mathrm{QCD}}$ ), which might leave open the possibility of large net contributions to other EDMs. However, for any fixed values of the $\mathrm{CP}$-conserving MCPMFV parameters, the compact ranges of the $\mathrm{CP}$-violating parameters imply that the value of any other CP-violating observable, e.g., an EDM, is necessarily bounded. The question then arises whether the prospective sensitivity of any proposed experiment reaches below the maximum value attainable in any given theoretical framework, such as the MCPMFV model (with or without the possibility that $\theta_{\mathrm{QCD}} \neq 0$ ). Clearly, any experiment that has insufficient sensitivity to search below the maximum value is not interesting for testing the MCPMFV model, whereas any experiment capable of reaching below the maximum value may either make a measurement or exclude part of the MCPMFV parameter space.

In a recent paper [12] we proposed a novel analytical technique, based on a differentialgeometrical construction (see also [13]), for finding the maximal values of CP-violating observables subject to the existing EDM constraints, which is exact in the linear approximation. We applied this technique to find maximal values of the EDMs of the Deuteron and 
muon, the CP-violating asymmetry in $b \rightarrow s \gamma$ decay, $A_{C P}$, and the $B_{s}$ mixing phase [12]. We found that, whereas the EDM of the Deuteron in the MCPMFV model (allowing also for $\theta_{\mathrm{QCD}} \neq 0$ ) might be one (two) orders of magnitude larger than the prospective experimental sensitivity, and $A_{C P}$ might also be detectable, the EDM of the muon and the contribution to the $B_{s}$ mixing phase in the MCPMFV model are likely to be too small to be observable in the near future.

In this paper we extend the applications of our analytical method $[12,13]$ to calculate the maximal values in the MCPMFV of the EDMs of some nuclei that are enhanced by the Schiff moment contributions associated with nearby parity-doublet states [14]. An experimental campaign is now being considered for HIE-ISOLDE that could search for EDMs of radium isotopes [15], accompanied by measurements of octupole collectivity in radium isotopes [16] that would be needed to interpret EDM measurements in terms of time-reversal violating interactions.

We find that values of the ${ }^{225} \mathrm{Ra}$ EDM that are considerably larger than $10^{-27} \mathrm{e} \cdot \mathrm{cm}$ (well within the estimated sensitivity of the proposed HIE-ISOLDE experiment) are possible in the MCPMFV model. How much larger depends quite sensitively on the implementation of the experimental constraint on the EDM of ${ }^{199} \mathrm{Hg}$. As discussed below, several theoretical calculations of the EDM are available, and most of them give very similar allowed ranges for the EDM of ${ }^{225} \mathrm{Ra}$, in the range 6 to $10 \times 10^{-27} e \cdot \mathrm{cm}$. However, one theoretical calculation of the ${ }^{199} \mathrm{Hg}$ EDM yields much weaker constraints on the CP-violating parameters of the MCPMFV, and hence yields a much larger maximal value of the ${ }^{225} \mathrm{Ra}$ EDM, namely $50 \times 10^{-27} e \cdot \mathrm{cm}$.

\section{$2 \quad$ Schiff moments for selected nuclei}

A CP-violating atomic EDM may arise from intrinsic EDMs of the constituent nucleons and atomic electrons, CP-odd electron-nucleon interactions, and the CP-odd nuclear moment known as the Schiff moment. The Schiff moments of several atoms have been calculated in the literature, and expressed in terms of sums of products of CP-even and CP-odd pionnucleon-nucleon $(\pi N N)$ couplings. We recall that the CP-even strong $\pi N N$ interaction is given by $[17]$

$$
\mathcal{L}_{\pi N N}^{\text {strong }}=g_{\pi N N}\left[\sqrt{2}\left(\bar{p} i \gamma_{5} n \pi^{+}+\bar{n} i \gamma_{5} p \pi^{-}\right)+\left(\bar{p} i \gamma_{5} p-\bar{n} i \gamma_{5} n\right) \pi^{0}\right],
$$

where $g_{\pi N N}=13.45$. On the other hand, the CP-odd (T-odd) $\pi N N$ interactions are given by $[17,18]$

$$
\begin{gathered}
\mathcal{L}_{\pi N N}^{\mathrm{T}}=\bar{g}_{\pi N N}^{(0)} \bar{N} \tau^{a} N \pi^{a}+\bar{g}_{\pi N N}^{(1)} \bar{N} N \pi^{0}+\bar{g}_{\pi N N}^{(2)}\left(\bar{N} \tau^{a} N \pi^{a}-3 \bar{N} \tau^{3} N \pi^{0}\right) \\
=\bar{g}_{\pi N N}^{(0)}\left[\sqrt{2}\left(\bar{p} n \pi^{+}+\bar{n} p \pi^{-}\right)+(\bar{p} p-\bar{n} n) \pi^{0}\right] \\
+\bar{g}_{\pi N N}^{(1)}(\bar{p} p+\bar{n} n) \pi^{0} \\
\quad+\bar{g}_{\pi N N}^{(2)}\left[\sqrt{2}\left(\bar{p} n \pi^{+}+\bar{n} p \pi^{-}\right)-2(\bar{p} p-\bar{n} n) \pi^{0}\right]
\end{gathered}
$$

in terms of the isoscalar $\bar{g}_{\pi N N}^{(0)}$, isovector $\bar{g}_{\pi N N}^{(1)}$, and isotensor $\bar{g}_{\pi N N}^{(2)}$ T-violating pionnucleon couplings. 


\begin{tabular}{|c|c|c|c|c|c|c|}
\hline Atom & Ref. & Interaction & $-a_{0}$ & $-a_{1}$ & $a_{2}$ & $-b$ \\
\hline \multirow{8}{*}{${ }^{199} \mathrm{Hg}$} & {$[20]$} & - & 0.0004 & 0.055 & 0.009 & - \\
\hline & \multirow[t]{2}{*}{ [24] } & $\mathrm{SkO}^{\prime}$ & 0.010 & 0.074 & 0.018 & - \\
\hline & & (average) & 0.007 & 0.071 & 0.018 & - \\
\hline & \multirow[t]{5}{*}{ [19] } & SLy4 (HF) & 0.013 & -0.006 & 0.022 & 0.003 \\
\hline & & SIII (HF) & 0.012 & 0.005 & 0.016 & 0.004 \\
\hline & & $\mathrm{SV}(\mathrm{HF})$ & 0.009 & -0.0001 & 0.016 & 0.002 \\
\hline & & SLy4 (HFB) & 0.013 & -0.006 & 0.024 & 0.007 \\
\hline & & $\mathrm{SkM}^{*}(\mathrm{HFB})$ & 0.041 & -0.027 & 0.069 & 0.013 \\
\hline \multirow{2}{*}{${ }^{225} \mathrm{Ra}$} & [25] & $\mathrm{SkO}^{\prime}$ (zero range) & -5.1 & 10.4 & -10.1 & - \\
\hline & {$[26]$} & $\mathrm{SkO}^{\prime}$ & -1.5 & 6.0 & -4.0 & - \\
\hline \multirow{4}{*}{${ }^{211} \mathrm{Rn}$} & {$[27]$} & & 0.019 & -0.061 & 0.053 & - \\
\hline & \multirow[t]{3}{*}{ [19] } & SLy4 & 0.042 & -0.018 & 0.071 & 0.016 \\
\hline & & $\mathrm{SkM}^{*}$ & 0.042 & -0.028 & 0.078 & 0.015 \\
\hline & & SIII & 0.034 & -0.0004 & 0.064 & 0.015 \\
\hline
\end{tabular}

Table 1. The coefficients $a_{i}, i=0,1,2$ and $b$ of the Schiff moments of ${ }^{199} \mathrm{Hg},{ }^{225} \mathrm{Ra}$, and ${ }^{211} \mathrm{Rn}$, expressed in units of $e \cdot \mathrm{fm}^{3}$. The labels HB and HFB stand for calculations in the Hartree-Fock and Hartree-Fock-Bogoliubov approximations, respectively: see ref. [19] for details. We have changed the signs of the coefficients $a_{0}, a_{1}$, and $b$ to follow the conventions of ref. [18].

The Schiff moment is linear in the CP-odd $\pi N N$ couplings $\bar{g}_{\pi N N}^{(i)}$, and may be written as [19]

$$
S=\left(a_{0}+b\right) g_{\pi N N} \bar{g}_{\pi N N}^{(0)}+a_{1} g_{\pi N N} \bar{g}_{\pi N N}^{(1)}+\left(a_{2}-b\right) g_{\pi N N} \bar{g}_{\pi N N}^{(2)} .
$$

Here the coefficients $a_{i}$ specify the dependence of the Schiff moment on the CP-odd interactions and the coefficient $b$ specifies its dependence on the nucleon dipole moments. The coefficients $a_{i}$ with $i=0,1,2$ and $b$ depend on the type of atom of interest, and some theoretical estimates for ${ }^{199} \mathrm{Hg},{ }^{225} \mathrm{Ra}$ and ${ }^{211} \mathrm{Rn}$, in units of $e \cdot \mathrm{fm}$ are collected in table 1 .

In the case of the ${ }^{199} \mathrm{Hg}$ Schiff moment, the results [20] adopted in [18, 21-23] are significantly different from the more recent calculations of $[19,24]$. In particular, the coefficient $a_{0}$ may be enhanced by a factor of $\sim 30$, whilst the coefficient $a_{1}$ may be reduced by a factor of $\sim 10$ and could even take the opposite sign. After considering the different numbers from the most recent calculations [19] in table 1, we have used the SIII (HF) calculation for our numerical illustration. As motivation, we note that the first SLy4 and the SV calculations do not yield the right quantum numbers for the ground state, that the second SLy4 calculation was found to be unable to project HFB states, and that the $\mathrm{SkM}^{*}$ calculation gives a spherical minimum.

The CP-odd couplings $\bar{g}_{\pi N N}^{(i)}$ may be generated by chromoelectric dipole moments (CEDMs) of the quarks and/or dimension-six four-fermion interactions. In ref. [28], one may find full expressions for the contributions to the CP-odd isoscalar $\bar{g}_{\pi N N}^{(0)}$ and isovector 
$\bar{g}_{\pi N N}^{(1)}$ couplings from the CEDMs of the light quarks: ${ }^{1}$

$$
\begin{aligned}
& \bar{g}_{\pi N N}^{(0)}=(-0.5 \text { to } 1.5) \times 10^{-12} \frac{\left(d_{u}^{C}+d_{d}^{C}\right) / g_{s}}{10^{-26} \mathrm{~cm}} \frac{|\langle\bar{q} q\rangle|}{(225 \mathrm{MeV})^{3}}, \\
& \bar{g}_{\pi N N}^{(1)}=2_{-1}^{+4} \times 10^{-12} \frac{\left(d_{u}^{C}-d_{d}^{C}\right) / g_{s}}{10^{-26} \mathrm{~cm}} \frac{|\langle\bar{q} q\rangle|}{(225 \mathrm{MeV})^{3}} .
\end{aligned}
$$

We note that a 'best value' is not available for $\bar{g}_{\pi N N}^{(0)}$. For definiteness, we have taken $\bar{g}_{\pi N N}^{(0)} / \bar{g}_{\pi N N}^{(1)}=0.2\left(d_{u}^{C}+d_{d}^{C}\right) /\left(d_{u}^{C}-d_{d}^{C}\right)$, as follows from assuming that the couplings $\bar{g}_{\pi N N}^{(0)}$ and $\bar{g}_{\pi N N}^{(1)}$ are proportional to the matrix elements of $\bar{u} u-\bar{d} d$ and $\bar{u} u+\bar{d} d$ in the nucleon state, respectively. It is known that the matrix element with the minus $(-)$ sign is smaller than the one with the plus $(+)$ sign by a a factor $\sim 5$ to $10[29]$.

We have also included the contribution of the dimension-six four-fermion interactions to the isovector coupling which can be enhanced for a large value of $\tan \beta[30,31]$ :

$$
\begin{aligned}
\bar{g}_{\pi N N}^{(1)} & =\frac{\langle\bar{q} q\rangle}{2 f_{\pi}}\left\langle N\left|C_{d d} \bar{d} d+C_{s d} \bar{s} s+C_{b d} \bar{b} b\right| N\right\rangle \\
& =-8 \times 10^{-3} \mathrm{GeV}^{3}\left[\frac{0.5 C_{d d}}{m_{d}}+3.3 \kappa \frac{C_{s d}}{m_{s}}+(1-0.25 \kappa) \frac{C_{b d}}{m_{b}}\right],
\end{aligned}
$$

where the couplings appearing in (2.5) are defined via the interaction Lagrangian

$$
\mathcal{L}_{4 \mathrm{f}}=\sum_{f, f^{\prime}} C_{f f^{\prime}}(\bar{f} f)\left(\bar{f}^{\prime} i \gamma_{5} f^{\prime}\right)
$$

and $\kappa \equiv\left\langle N\left|m_{s} \bar{s} s\right| N\right\rangle / 220 \mathrm{MeV} \simeq 0.50 \pm 0.25$. The contribution of the four-fermion interactions to the isoscalar coupling $\bar{g}_{\pi N N}^{(0)}$ is ignored and the isotensor coupling $\bar{g}_{\pi N N}^{(2)}$, which changes the isospin by two units, is neglected in this work (as in ref. [28]), since it can be generated only at the expense of an additional $m_{u}-m_{d}$ suppression.

In the following, we concentrate on the EDMs of ${ }^{199} \mathrm{Hg}$ and ${ }^{225} \mathrm{Ra}$ atoms, because there is already a stringent experimental upper limit on the former, and there is a proposal to measure the latter at HIE-ISOLDE [15]. Moreover, we note that the estimates of the contributions to the Schiff moment of ${ }^{211} \mathrm{Rn}$ shown in table 1 are considerably less favourable.

The experimental constraint on the EDM of ${ }^{199} \mathrm{Hg}[32,33]$,

$$
\left|d_{\mathrm{Hg}}^{\mathrm{EXP}}\right|<3.1 \times 10^{-29} e \cdot \mathrm{cm}(95 \% \text { C.L. }),
$$

is among the most stringent constraints on $\mathrm{CP}$ violation imposed by atomic EDM experiments. There are several calculations of the ${ }^{199} \mathrm{Hg}$ EDM in terms of the Schiff moment in the literature, which can be cast in the general form

$$
d_{\mathrm{Hg}}[S]=10^{-17} \mathcal{C}_{\mathrm{Hg}}^{S} e \cdot \mathrm{cm} \times\left(\frac{S}{e \cdot \mathrm{fm}^{3}}\right),
$$

with the coefficient taking the values $\mathcal{C}_{\mathrm{Hg}}^{S}=-4[34,35],-2.8[36]$ and +5.07 [37]. We note that we have changed the sign of the most recent calculation based on relativistic coupledcluster theory [37], so as to match the conventions in the previous calculations [34-36]

\footnotetext{
${ }^{1}$ We adopt the conventions and notations of [21-23].
} 
based on coupled perturbed Hartree-Fock calculations, and that it has a different sign. For our numerical calcluatons we take the estimates of the coefficient $\mathcal{C}_{\mathrm{Hg}}^{S}$ that have negative signs. To be specific, we have used the following four calculations of the Mercury EDM:

- From ref. [18],

$$
d_{\mathrm{Hg}}^{\mathrm{I}}[S] \simeq 1.8 \times 10^{-3} e \bar{g}_{\pi N N}^{(1)} / \mathrm{GeV},
$$

- Taking $\mathcal{C}_{\mathrm{Hg}}^{S}=-2.8$ [36] and the coefficients from ref. [20],

$$
d_{\mathrm{Hg}}^{\mathrm{II}}[S] \simeq 7.6 \times 10^{-6} e \bar{g}_{\pi N N}^{(0)} / \mathrm{GeV}+1.0 \times 10^{-3} e \bar{g}_{\pi N N}^{(1)} / \mathrm{GeV},
$$

- Taking $\mathcal{C}_{\mathrm{Hg}}^{S}=-2.8$ [36] and the average coeffcients from ref. [24],

$$
d_{\mathrm{Hg}}^{\mathrm{III}}[S] \simeq 1.3 \times 10^{-4} e \bar{g}_{\pi N N}^{(0)} / \mathrm{GeV}+1.4 \times 10^{-3} e \bar{g}_{\pi N N}^{(1)} / \mathrm{GeV},
$$

- Taking $\mathcal{C}_{\mathrm{Hg}}^{S}=-2.8$ [36] the $\mathrm{SIII}(\mathrm{HF})$ coeffcients from ref. [19],

$$
d_{\mathrm{Hg}}^{\mathrm{IV}}[S] \simeq 3.1 \times 10^{-4} e \bar{g}_{\pi N N}^{(0)} / \mathrm{GeV}+9.5 \times 10^{-5} e \bar{g}_{\pi N N}^{(1)} / \mathrm{GeV} .
$$

Compared to the expression $d_{\mathrm{Hg}}^{\mathrm{I}}[S]$ used in refs. [21-23], $d_{\mathrm{Hg}}^{\mathrm{II}}[S]$ is slightly smaller, and has a negligible isoscalar contribution, which is actually ignored in $d_{\mathrm{Hg}}^{\mathrm{I}}[S]$. Whilst the isoscalar contribution is still small in $d_{\mathrm{Hg}}^{\mathrm{III}}[S]$, it can be larger than the isovector contribution by a factor $\sim 3$ in $d_{\mathrm{Hg}}^{\mathrm{IV}}[S]$. However, we observe that $\left|d_{\mathrm{Hg}}^{\mathrm{IV}}[S]\right|$ is smaller than $\left|d_{\mathrm{Hg}}^{\mathrm{I}}[S]\right|$ by about an order of magnitude.

On the other hand, the EDM of ${ }^{225} \mathrm{Ra}$ is related to its Schiff moment by [36]

$$
d_{\mathrm{Ra}}[S]=-8.5 \times 10^{-17} e \cdot \mathrm{cm} \times\left(\frac{S}{e \cdot \mathrm{fm}^{3}}\right) .
$$

Taking the coefficients given in ref. [26], see table 1, we obtain

$$
d_{\mathrm{Ra}}[S] \simeq-8.7 \times 10^{-2} e \bar{g}_{\pi N N}^{(0)} / \mathrm{GeV}+3.5 \times 10^{-1} e \bar{g}_{\pi N N}^{(1)} / \mathrm{GeV} .
$$

We note that the $\bar{g}_{\pi N N}^{(1)}$ contribution the EDM of ${ }^{225} \mathrm{Ra}$ is about 200 times larger than to the Mercury EDM $d_{\mathrm{Hg}}^{\mathrm{I}}[S]$, an enhancement due to the existence of a nearby parity-doublet states [25].

\section{Differential-geometrical optimization method}

We briefly review our powerful analytical approach for finding the optimal choice of CPodd phases which maximize the size of a given $\mathrm{CP}$-violating observable $O$, while remaining compatible with the present EDM constraints [13]. We have applied this approach previously to estimate maximal values of the Deuteron and muon EDMs, the CP asymmetry in $b \rightarrow s \gamma$, and the phase in $B_{s}$ mixing [12], and it may be applied similarly to the case where the observable $O$ is the EDM of ${ }^{225}$ Ra. 
We consider a theory such as the MCPMFV SUSY model with six CP-odd phases, $\mathbf{\Phi}$, represented by a $6 \mathrm{D}$ phase vector, subject to three EDM constraints denoted by $E^{a, b, c}=0$, corresponding to the non-observation of the Thallium, neutron and Mercury EDMs. For any given value of the $\mathrm{CP}$-conserving parameters in the MCPMFV model, we may expand these EDMs and the observable $O$ in the small-phase approximation, defining the four $6 \mathrm{D}$ vectors $\mathbf{E}^{a, b, c}=\nabla E^{a, b, c}$ and $\mathbf{O}=\nabla O$, and we assume that the four vectors $\mathbf{E}^{a, b, c}$ and $\mathbf{O}$ are linearly independent.

We then introduce the triple exterior product

$$
A_{\alpha \beta \gamma} \equiv E_{[\alpha}^{a} E_{\beta}^{b} E_{\gamma]}^{c}
$$

where the Greek indices label the components of the vectors in the $6 \mathrm{D}$ space, i.e., $\alpha, \beta, \gamma=$ $1,2, \ldots, 6$. The square brackets on the r.h.s. of (3.1) indicate that the tensor $A_{\alpha \beta \gamma}$ is obtained by fully antisymmetrizing the vectors $E_{\alpha}^{a}, E_{\beta}^{b}$ and $E_{\gamma}^{c}$ in the indices $\alpha, \beta, \gamma$, i.e., $A_{\alpha \beta \gamma}=-A_{\beta \alpha \gamma}=-A_{\alpha \gamma \beta}$, etc. Borrowing a term from the calculus of differential forms, $A_{\alpha \beta \gamma}$ is a 3 -form. We also introduce the 2 -form

$$
B_{\mu \nu}=\varepsilon_{\mu \nu \lambda \rho \sigma \tau} O_{\lambda} E_{\rho}^{a} E_{\sigma}^{b} E_{\tau}^{c},
$$

where summation over repeated indices is implied and $\varepsilon_{\mu \nu \lambda \rho \sigma \tau}$ is the usual Levi-Civita tensor generalized to $6 \mathrm{D}$. In the language of differential forms, $B_{\mu \nu}$ is, up to an irrelevant overall factor, the Hodge-dual product between the 1-form $O_{\lambda}$, representing the CP-violating observable, and the 3 -form $A_{\alpha \beta \gamma}$.

The components $\Phi_{\alpha}^{*}$ of the optimal EDM-free direction maximizing $O$ can now be obtained from the Hodge-dual product of the 3 -form $A_{\beta \gamma \delta}$ and the 2-form $B_{\mu \nu}$. Explicitly,

$$
\Phi_{\alpha}^{*}=\mathcal{N} \varepsilon_{\alpha \beta \gamma \delta \mu \nu} A_{\beta \gamma \delta} B_{\mu \nu}=\mathcal{N} \varepsilon_{\alpha \beta \gamma \delta \mu \nu} \varepsilon_{\mu \nu \lambda \rho \sigma \tau} E_{\beta}^{a} E_{\gamma}^{b} E_{\delta}^{c} O_{\lambda} E_{\rho}^{a} E_{\sigma}^{b} E_{\tau}^{c},
$$

where we have included an unknown overall normalization factor $\mathcal{N}$. By construction, the $6 \mathrm{D}$ phase vector $\boldsymbol{\Phi}^{*}$ is orthogonal to the three vectors $\mathbf{E}^{a, b, c}$, and therefore satisfies the desired EDM constraints, $E^{a}=E^{b}=E^{c}=0$, in the small-phase approximation. We observe that the magnitude $\phi^{*} \equiv\left|\Phi^{*}\right|$, and hence the overall normalization factor $\mathcal{N}$, can only be determined by a numerical analysis of the actual experimental limits on the three EDMs. As in the 3D example, the maximum allowed value of the CP-violating observable $O$ is given in the small-phase approximation by

$$
O=\phi^{*} \widehat{\Phi}_{\kappa}^{*} O_{\kappa}= \pm \mathcal{N}\left|\varepsilon_{\mu \nu \alpha \beta \gamma \delta} \varepsilon_{\mu \nu \lambda \rho \sigma \tau} O_{\alpha} O_{\lambda} E_{\beta}^{a} E_{\gamma}^{b} E_{\delta}^{c} E_{\rho}^{a} E_{\sigma}^{b} E_{\tau}^{c}\right|
$$

where the caret denotes the components of a unit-norm vector. As discussed in [12], quadratic and higher-order derivative terms with respect to the CP-odd phases will generically prefer a particular sign for the optimal value of $O$.

We can also allow for the possible presence of a non-zero strong CP phase $\theta_{\mathrm{QCD}}$ in the theory, in which case the corresponding CP-odd phase vector $\boldsymbol{\Phi}$ becomes seven-dimensional (7D) in the MCPMFV SUSY model. The generalization of the above construction of the optimal value of the observable $O$ is discussed in section 5 of [12]. 


\section{Results}

In this section we use the above the differential-geometrical approach to analyze the maximal value of the ${ }^{225} \mathrm{Ra}$ EDM obtainable in CP-violating variants of the following representative CMSSM benchmark scenario which predicts the mass spectrum of SUSY particles in the sub-TeV region:

$$
\begin{aligned}
& \left|M_{1,2,3}\right|=350 \mathrm{GeV}, \\
& M_{H_{u}}^{2}=M_{H_{d}}^{2}=\widetilde{M}_{Q}^{2}=\widetilde{M}_{U}^{2}=\widetilde{M}_{D}^{2}=\widetilde{M}_{L}^{2}=\widetilde{M}_{E}^{2}=(100 \mathrm{GeV})^{2}, \\
& \left|A_{u}\right|=\left|A_{d}\right|=\left|A_{e}\right|=100 \mathrm{GeV},
\end{aligned}
$$

at the GUT scale, introducing non-zero $\mathrm{CP}$-violating phases and varying $\tan \beta$ ( $\left.M_{\mathrm{SUSY}}\right)$. We adopt the convention that $\Phi_{\mu}=0^{\circ}$, and we vary independently the following six MCPMFV phases at the GUT scale: $\Phi_{1}, \Phi_{2}, \Phi_{3}, \Phi_{A_{u}}, \Phi_{A_{d}}$, and $\Phi_{A_{e}}$. In the 7D case, in addition to the $6 \mathrm{CP}$ phases, we consider a non-zero strong $\mathrm{CP}$ phase $\theta_{\mathrm{QCD}}$. The scenario (4.1) considered in this work is similar to one with $\left|M_{1,2,3}\right|=250 \mathrm{GeV}$ that we considered previously $[3,12,21]$. The somewhat larger value $\left|M_{1,2,3}\right|=350 \mathrm{GeV}$ is chosen here for consistency with the recent SUSY search results reported by the CMS Collaboration at the LHC [38]. When $\tan \beta=10, \Phi_{1,2,3}=0^{\circ}$, and $\Phi_{A_{u}, A_{d}, A_{e}}=180^{\circ}$, the previous scenario with $\left|M_{1,2,3}\right|=250 \mathrm{GeV}$ became the well-known SPS1a point [39-42] (also known as benchmark B). The scenario (4.1) is more similar to benchmarks C, G and I of [3942] when $\tan \beta=10,20$ or 35 , respectively. Our calculations of the EDMs are based on refs. [12, 21], which include the two-loop diagrams mediated by the $\gamma-H^{ \pm}-W^{\mp}$ and $\gamma-W^{ \pm_{-}}$ $W^{\mp}$ couplings, and we take into account the effects of the different computations of the Schiff moment of the Mercury nucleus as explained in section 2.

\subsection{The MCPMFV SUSY model with 6 CP phases}

In order to analyze the scenario (4.1), we first make Taylor expansions of the following four EDMs in terms of the MCPMFV CP-violating phases:

$$
d_{\mathrm{Tl}} / d_{\mathrm{Tl}}^{\mathrm{EXP}}, \quad d_{\mathrm{n}} / d_{\mathrm{n}}^{\mathrm{EXP}}, \quad d_{\mathrm{Hg}} / d_{\mathrm{Hg}}^{\mathrm{EXP}}, \quad d_{\mathrm{Ra}} /\left(10^{-27} e \cdot \mathrm{cm}\right),
$$

where we choose the following normalization factors:

$$
d_{\mathrm{Tl}}^{\mathrm{EXP}}=9 \times 10^{-25} e \cdot \mathrm{cm}, \quad d_{\mathrm{n}}^{\mathrm{EXP}}=3 \times 10^{-26} e \cdot \mathrm{cm}, \quad d_{\mathrm{Hg}}^{\mathrm{EXP}}=3.1 \times 10^{-29} e \cdot \mathrm{cm},
$$

which correspond to the current experimental limits on the EDMs of Thallium [43], the neutron [44], and Mercury [32, 33]. The normalization factor for the ${ }^{225}$ Ra EDM, namely $10^{-27} e \cdot \mathrm{cm}$, is typical of the estimated experimental sensitivity.

In figure 1, we show the absolute values of the components of the three $6 \mathrm{D}$ MCPMFV vectors characterizing the existing EDM constraints and the $6 \mathrm{D}$ vector representing the $d_{\mathrm{Ra}}$ observable, for the scenario (4.1) varying $\tan \beta$ in a small-phase expansion around the CP-conserving point $\Phi_{1}=\Phi_{2}=\Phi_{3}=\Phi_{A_{u}}=\Phi_{A_{d}}=\Phi_{A_{e}}=0^{\circ}{ }^{2}{ }^{2}$ The solid lines represent

\footnotetext{
${ }^{2}$ We also display (as magenta lines) the corresponding components for 7 th components corresponding to the QCD phase $\bar{\theta}$, which we discuss later.
} 

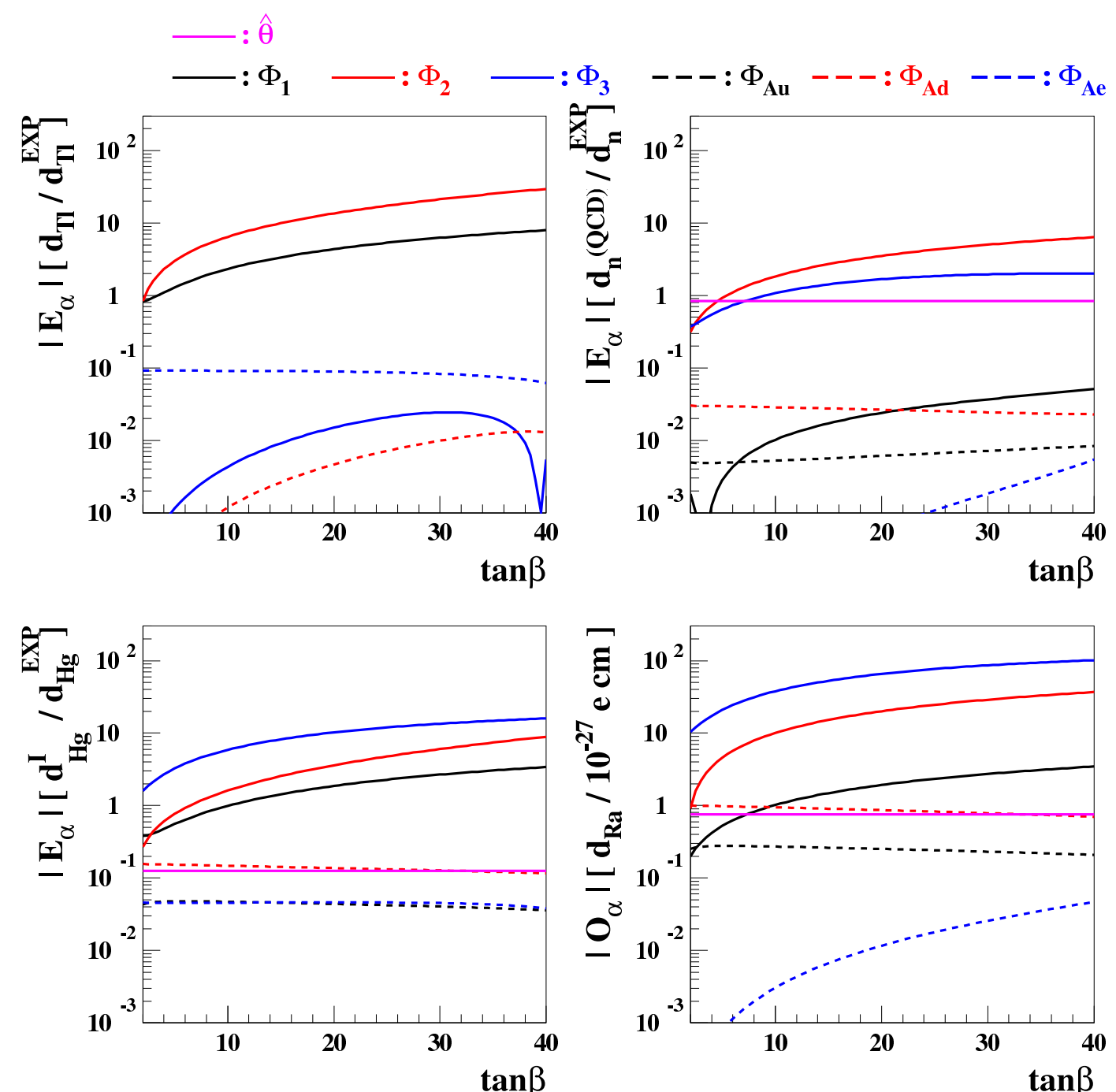

Figure 1. The absolute values of the components of the three vectors $\mathbf{E}_{\alpha}$ of MCPMFV CPviolating phases representing the present EDM constraints on $d_{\mathrm{Tl}}$ (upper left), $d_{\mathrm{n}}$ (upper right), and $d_{\mathrm{Hg}}$ (lower left), and those of the vector representing the EDM of ${ }^{225} \mathrm{Ra}$ (lower right) in smallphase expansions around the CP-conserving point, as functions of $\tan \beta$ for the scenario (4.1). We use the computation employing the QCD sum rule technique and the estimate $d_{\mathrm{Hg}}^{\mathrm{I}}(2.9)$ for the neutron and Mercury EDMs, respectively. The CP-violating phases are measured in degrees.

the $\mathrm{CP}$-violating phases of the gaugino mass parameters, and the dashed lines the trilinear $A$ parameters, respectively. The components corresponding to the $\mathrm{CP}$-violating gaugino phases dominate in all cases, increasingly as $\tan \beta$ grows, with the exception of the $\Phi_{3}$ component of $\mathbf{E}^{\mathrm{d}_{\mathrm{Tl}}}$. For example, when $\tan \beta=40,\left|d_{\mathrm{Tl}}\right|$ and $\left|d_{\mathrm{n}}\right|$ are larger than the current experimental limits by factors $\sim 30$ and $\sim 5$, respectively, even when $\Phi_{2}=1^{\circ}$, whereas $\left|d_{\mathrm{Hg}}^{\mathrm{I}}\right|$ is larger than the current limit by a factor $\sim 15(\sim 8)$ when $\Phi_{3}=1^{\circ}\left(\Phi_{2}=1^{\circ}\right){ }^{3}$ We see that $\left|d_{\text {Ra }}\right|$ can be as large as $\sim 100 \times 10^{-27} e \mathrm{~cm}\left(\sim 40 \times 10^{-27} e \mathrm{~cm}\right)$ if $\Phi_{3}=1^{\circ}\left(\Phi_{2}=1^{\circ}\right)$.

\footnotetext{
${ }^{3}$ Throughout this work, the CP-violating phases are measured in units of degrees.
} 


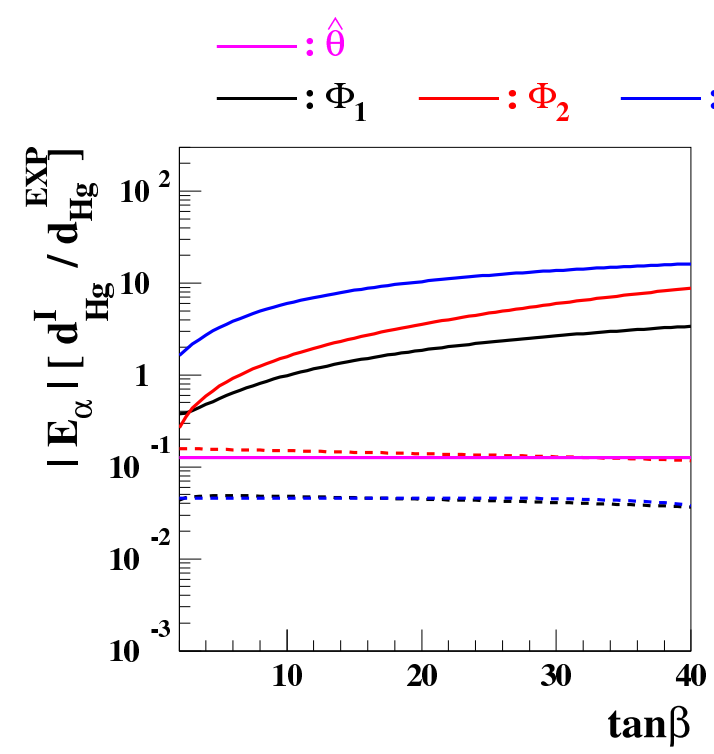

$: \Phi_{3}---: \Phi_{\mathrm{Au}}---: \Phi_{\mathrm{Ad}}---: \Phi_{\mathrm{Ae}}$
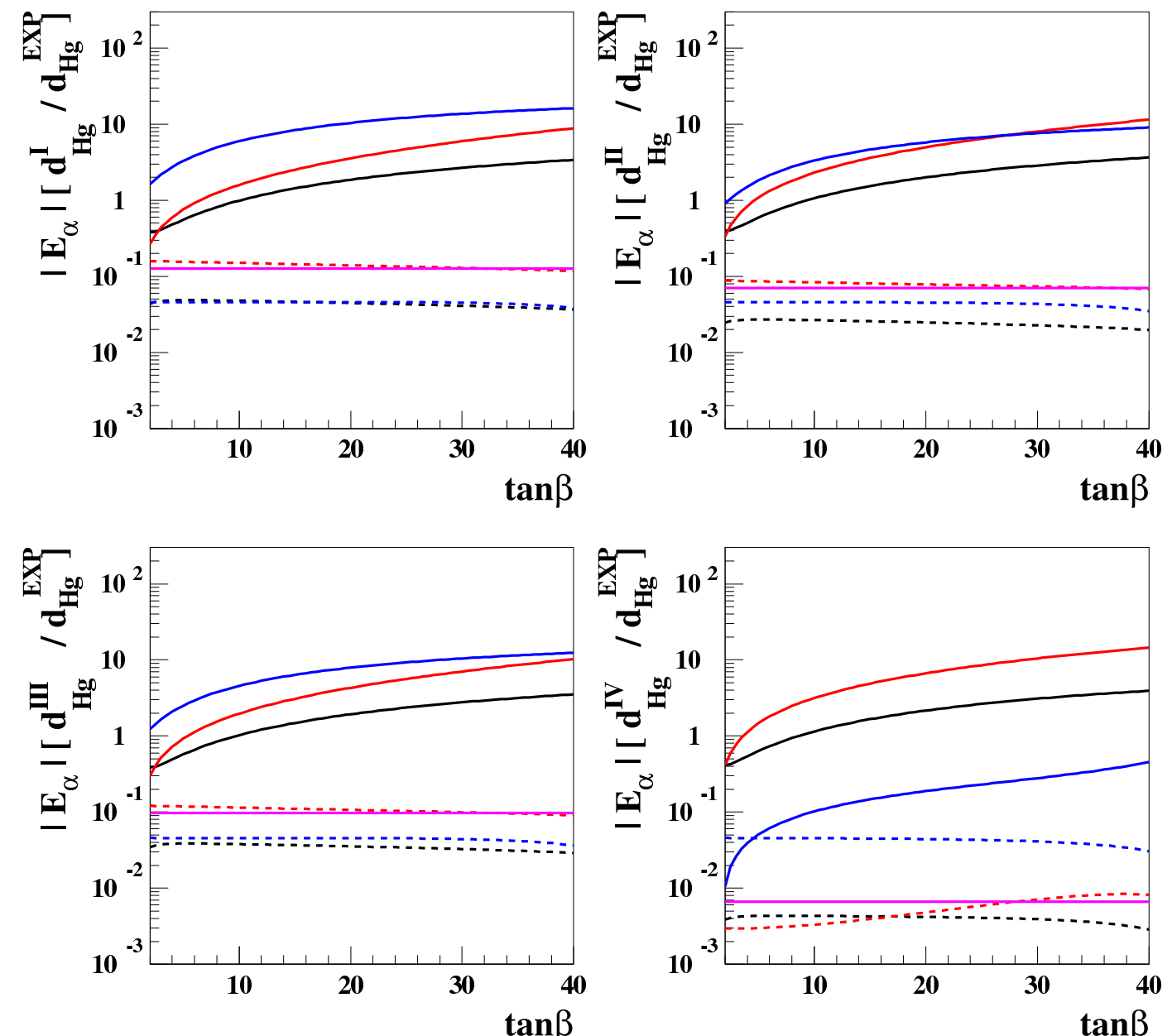

Figure 2. The absolute values of the components of the $6 \mathrm{D}$ vector $\mathbf{E}_{\alpha}$ of MCPMFV CP-violating phases representing the present EDM constraints on the Mercury EDM, as obtained using the four different calculations of $d_{\mathrm{Hg}}$ discussed in section 2: $d_{\mathrm{Hg}}^{\mathrm{I}}$ (upper left), $d_{\mathrm{Hg}}^{\mathrm{II}}$ (upper right), $d_{\mathrm{Hg}}^{\mathrm{III}}$ (lower right), and $d_{\mathrm{Hg}}^{\mathrm{IV}}$ (lower left). The line styles are the same as in figure 1 . The CP-violating phases are measured in degrees.

The relative contributions of the different $\mathrm{CP}$-violating MCPMFV phases to $d_{\mathrm{Hg}}$ vary according to the choice of theoretical calculation, as shown in figure $2 .{ }^{4}$ Specifically we observe the $\Phi_{3}$ contribution exhibits strong variations, and that it is much suppressed if the $\left|d_{\mathrm{Hg}}^{\mathrm{IV}}\right|$ calculation is used.

In figure 3 we show the cosines of the angles between the 6D MCPMFV vector representing the observable $\mathbf{O}^{d_{\mathrm{Ra}}}$ and the EDM vectors, which are defined by

$$
C_{\mathbf{O}, \mathbf{E}^{a}}=\frac{\mathbf{O} \cdot \mathbf{E}^{a}}{|\mathbf{O}|\left|\mathbf{E}^{a}\right|}
$$

\footnotetext{
${ }^{4}$ We again display (as magenta lines) the corresponding components for possible 7 th components corresponding to the QCD phase $\bar{\theta}$, which we discuss later.
} 


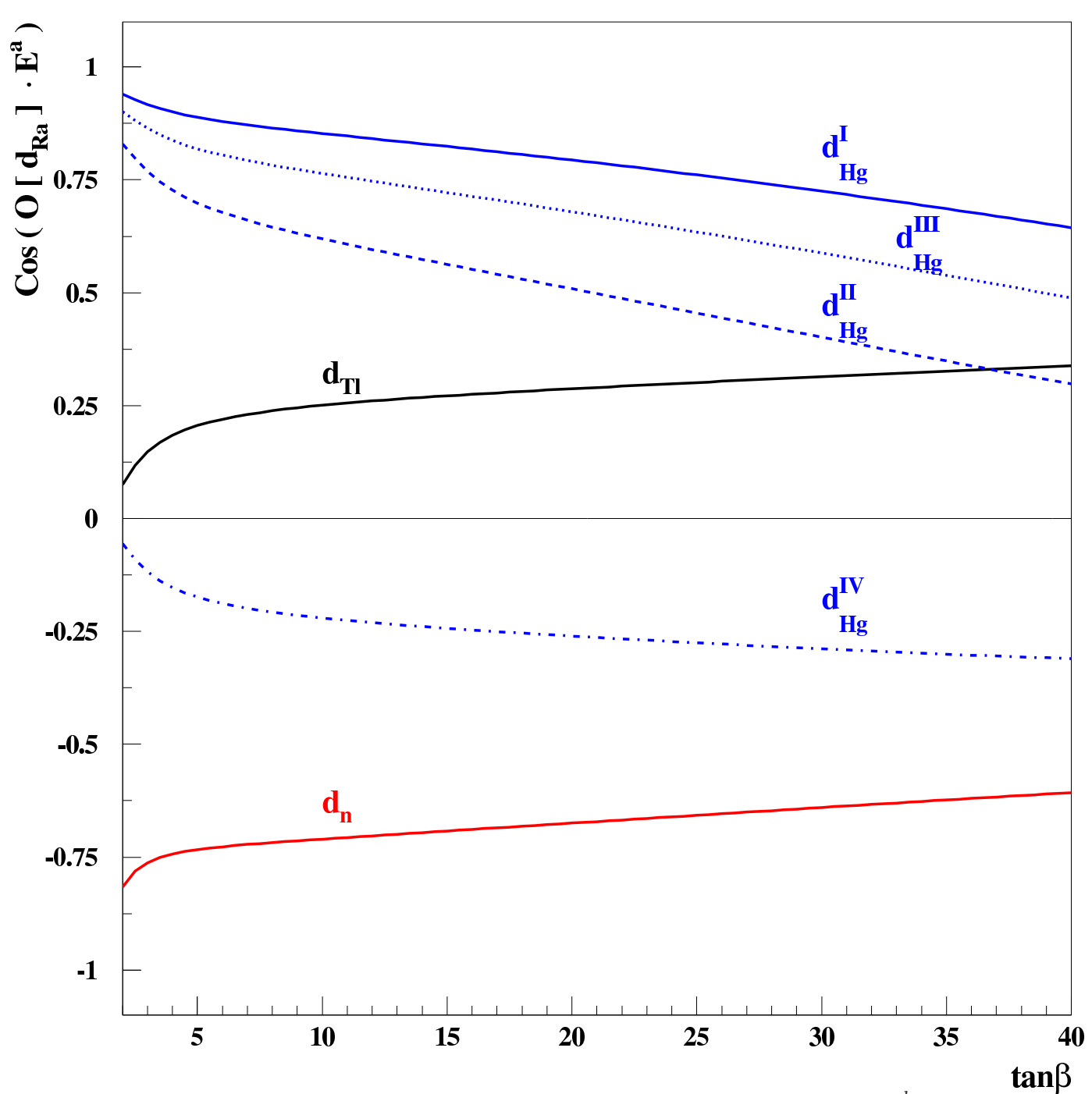

Figure 3. The cosines of the angles between the observable $6 \mathrm{D}$ vector $\mathbf{O}^{d_{\mathrm{Ra}}}$ and the EDMconstraint $6 \mathrm{D}$ vectors $\mathbf{E}^{d_{\mathrm{Tl}}}$ (black solid), $\mathbf{E}^{d_{\mathrm{n}}}$ (red solid), $\mathbf{E}^{d_{\mathrm{Hg}}^{\mathrm{I}}}$ (blue solid), $\mathbf{E}^{d_{\mathrm{Hg}}^{\mathrm{II}}}$ (blue dashed), $\mathbf{E}^{d_{\mathrm{Hg}}^{\mathrm{III}}}$ (blue dotted), and $\mathbf{E}^{d_{\mathrm{Hg}}^{\mathrm{IV}}}$ (blue dash-dotted) as functions of $\tan \beta$.

These quantities display the degrees of degeneracy, or alignment, between the observable $\mathrm{O}^{d_{\mathrm{Ra}}}$ and the EDM vectors $E^{a}$. We see that the alignments between $d_{\mathrm{Ra}}$ and $d_{\mathrm{Tl}}$ and $d_{\mathrm{Hg}}^{\mathrm{IV}}$ are quite small. On the other hand, the alignments between $d_{\mathrm{Ra}}$ and $d_{\mathrm{Hg}}^{\mathrm{I}, \mathrm{II}, \mathrm{III}}$ are larger, though decreasing as $\tan \beta$ grows. This can be understood by observing that the additional contributions to the Mercury EDM, beyond those from the couplings $\bar{g}_{\pi N N}^{(0),(1)}$, increase with $\tan \beta$.

Having the vectors representing the EDM constraints and the observable $d_{\mathrm{Ra}}$ in hand, we now combine them to construct the optimal directions in the $6 \mathrm{D}$ space of CP-violating MCPMFV phases, using (3.3), so as to maximize $d_{\mathrm{Ra}}$ in the linear approximation. For comparison, we also consider two reference directions, which have $\Phi_{1}=\Phi_{A_{e}}=0$ and $\Phi_{2}=\Phi_{3}=0$, respectively. These two reference directions can be constructed by defining

$$
\Phi_{\alpha}^{*} \equiv \mathcal{N} \varepsilon_{\alpha \beta \gamma \delta \mu \nu} E_{\beta}^{a} E_{\gamma}^{b} E_{\delta}^{c} N_{\mu}^{(1)} N_{\nu}^{(2)},
$$



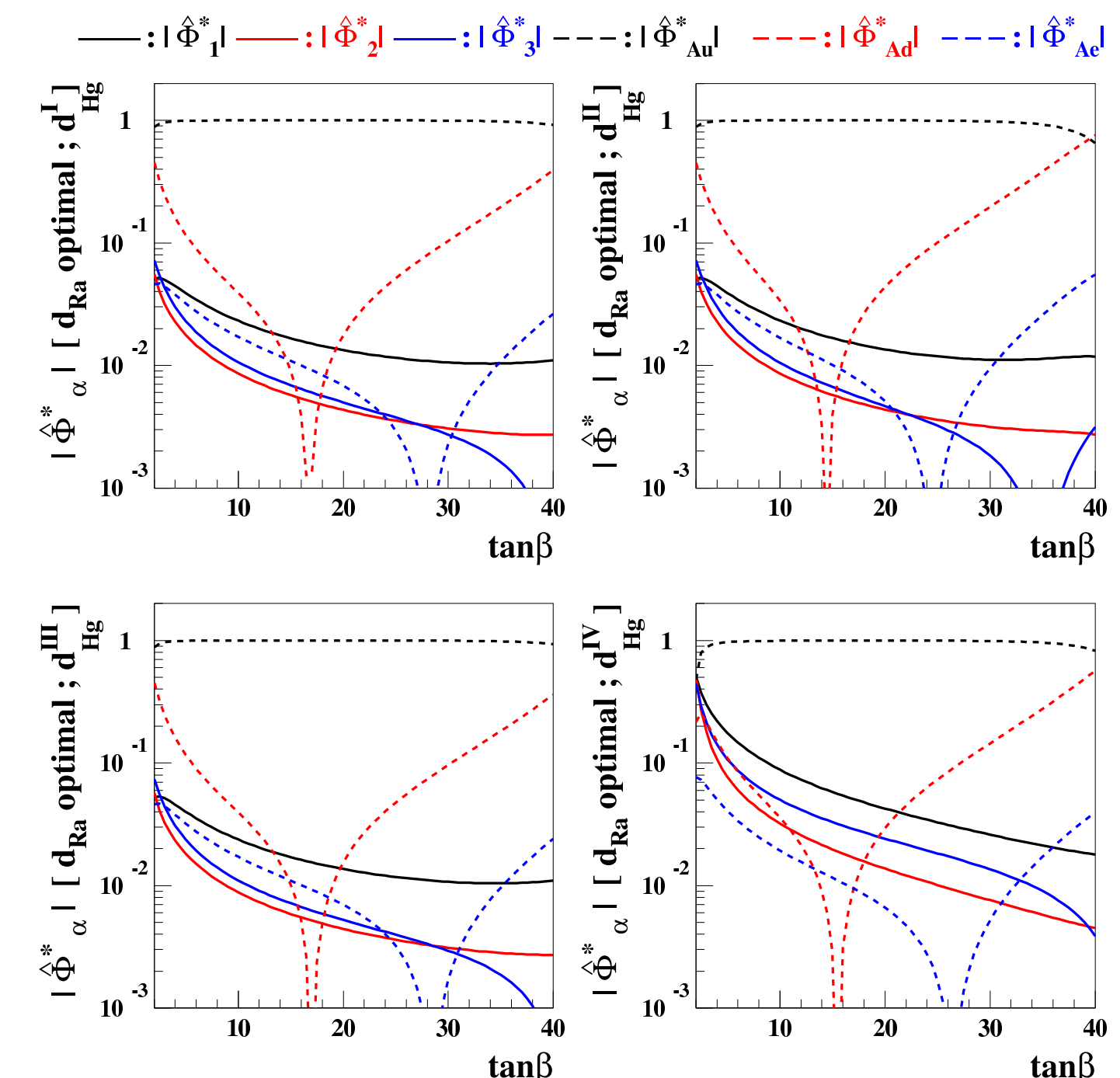

Figure 4. The absolute values of the six components of the normalized vectors in the directions where the size of $d_{\mathrm{Ra}}$ is optimized, as obtained using the calculations $d_{\mathrm{Hg}}^{\mathrm{I}}$ (upper left), $d_{\mathrm{Hg}}^{\mathrm{II}}$ (upper right), $d_{\mathrm{Hg}}^{\mathrm{III}}$ (lower right), and $d_{\mathrm{Hg}}^{\mathrm{IV}}$ (lower left) for the Mercury EDM. The CP-violating phases are measured in degrees.

where, for each direction, the two null directions $N_{\mu}^{(1,2)}$ are chosen as

$$
N_{\mu}^{(1)}=(1,0,0,0,0,0), \quad N_{\mu}^{(2)}=(0,0,0,0,0,1)
$$

for the direction $\Phi_{1}=\Phi_{A_{e}}=0$, and

$$
N_{\mu}^{(1)}=(0,1,0,0,0,0), \quad N_{\mu}^{(2)}=(0,0,1,0,0,0)
$$

for the direction $\Phi_{2}=\Phi_{3}=0$.

We display in figure 4 the absolute values of the six components of the normalized optimal vectors in the direction along which $d_{\mathrm{Ra}}$ is maximized, as obtained using $d_{\mathrm{Hg}}^{\mathrm{I}}$ (upper left), $d_{\mathrm{Hg}}^{\mathrm{II}}$ (upper right), $d_{\mathrm{Hg}}^{\mathrm{III}}$ (lower right), and $d_{\mathrm{Hg}}^{\mathrm{IV}}$ (lower left) for the Mercury EDM. We first observe that the $\Phi_{1,2,3}$ components (solid lines) are relatively small, and 


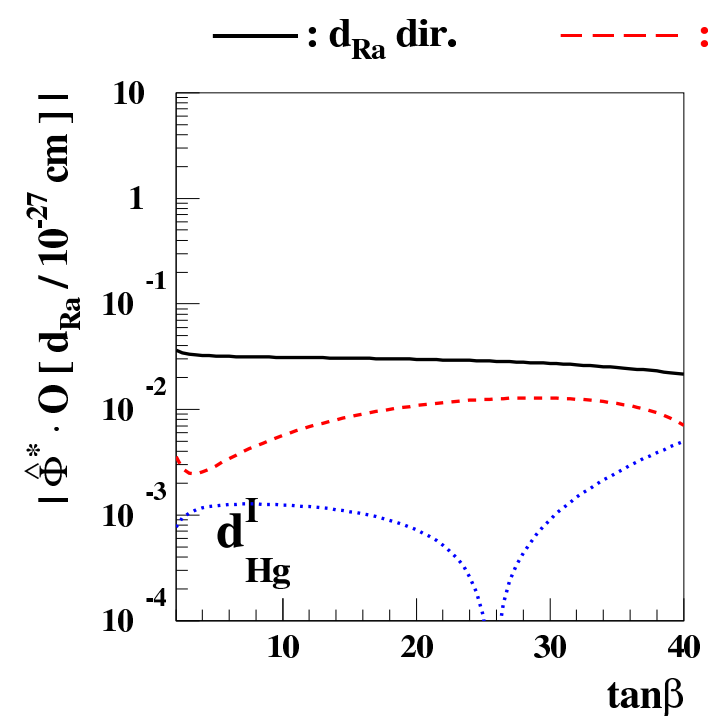

$\Phi_{1, \mathrm{Ae}}=\mathbf{0}$ dir. $\quad \cdots \cdots: \Phi_{2,3}=\mathbf{0}$ dir.
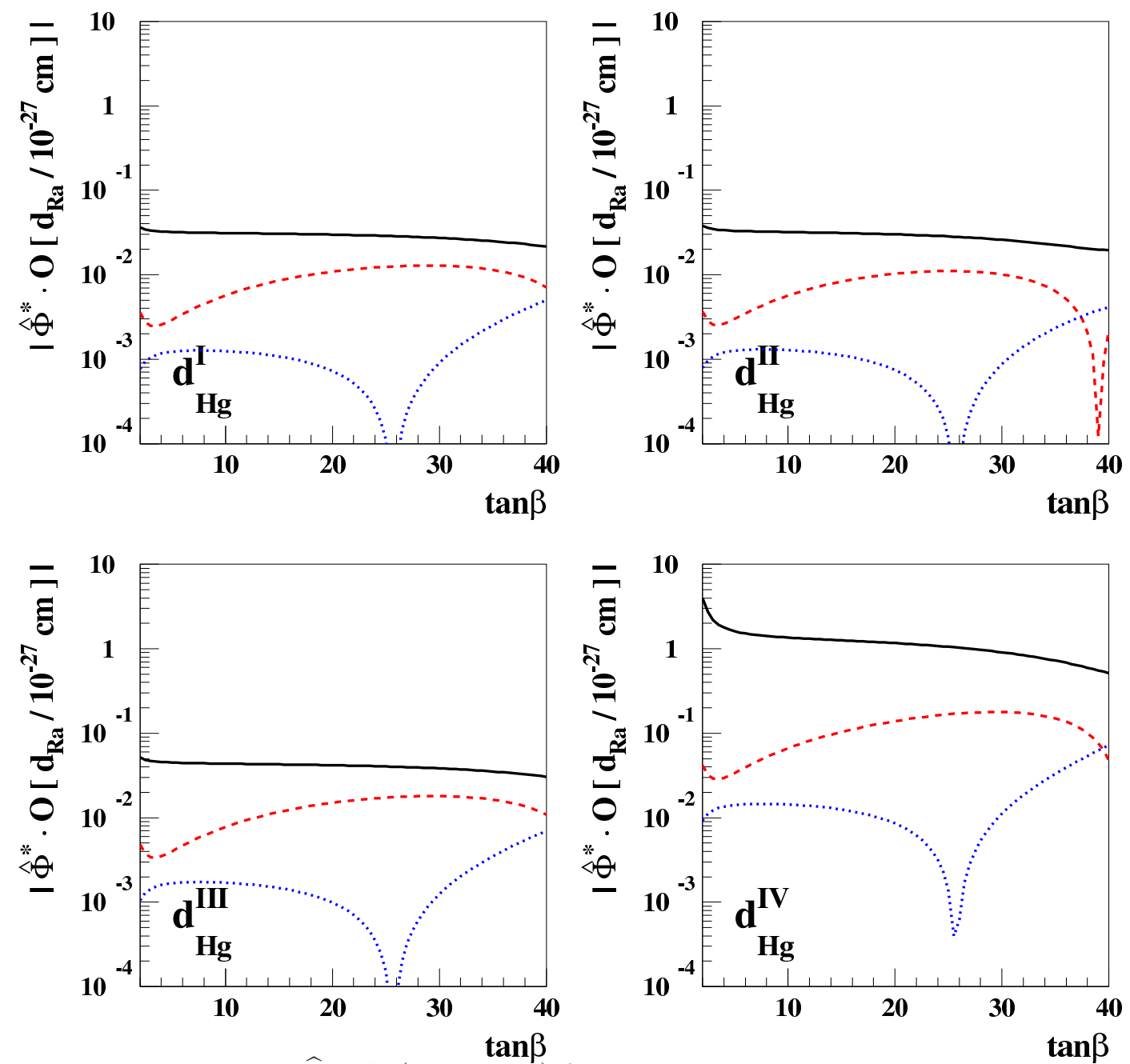

Figure 5. The products $\widehat{\Phi}^{*}$. O (black solid) for the optimal $d_{\mathrm{Ra}}$ direction, as obtained using the calculations $d_{\mathrm{Hg}}^{\mathrm{I}}$ (upper left), $d_{\mathrm{Hg}}^{\mathrm{II}}$ (upper right), $d_{\mathrm{Hg}}^{\mathrm{III}}$ (lower right), and $d_{\mathrm{Hg}}^{\mathrm{IV}}$ (lower left) for the Mercury EDM. For comparison purposes, we also show the products along the two reference directions with $\Phi_{1, A_{e}}=0$ (red dashed) and $\Phi_{2,3}=0$ (blue dotted).

decrease as $\tan \beta$ increases. Hence, all the optimal directions are mostly given by some combination of $\Phi_{A_{u}}$ (black dashed line) and $\Phi_{A_{d}}$ (red dashed line) directions implying, for $\tan \beta=40$, that $\left(\Phi_{A_{u}, A_{d}}\right)^{\max } \sim \phi^{*}$ whereas $\left(\Phi_{1,2,3}\right)^{\max } \sim \phi^{*} \times 10^{-2}$, as will be shown in the following.

In figure 5, we consider the products $\widehat{\Phi}^{*} \cdot \mathbf{O}$ of the $6 \mathrm{D}$ vectors in the normalized optimal directions for $d_{\mathrm{Ra}}$, and the Radium EDM, taking account of the uncertainty of the Mercury EDM calculation. The products determine the sizes of $d_{\mathrm{Ra}}$ along its optimal direction through the relations given in (3.4) when $\phi^{*}=1^{\circ}$. As shown below, $\phi^{*}$ could be as large as $\sim 100^{\circ}$ before the small-phase approximation breaks down and one of the three EDM constraints is violated. We observe that the direction constructed using the geometric prescription given in section 3 indeed gives the larger values of $d_{\mathrm{Ra}}$ than do the 


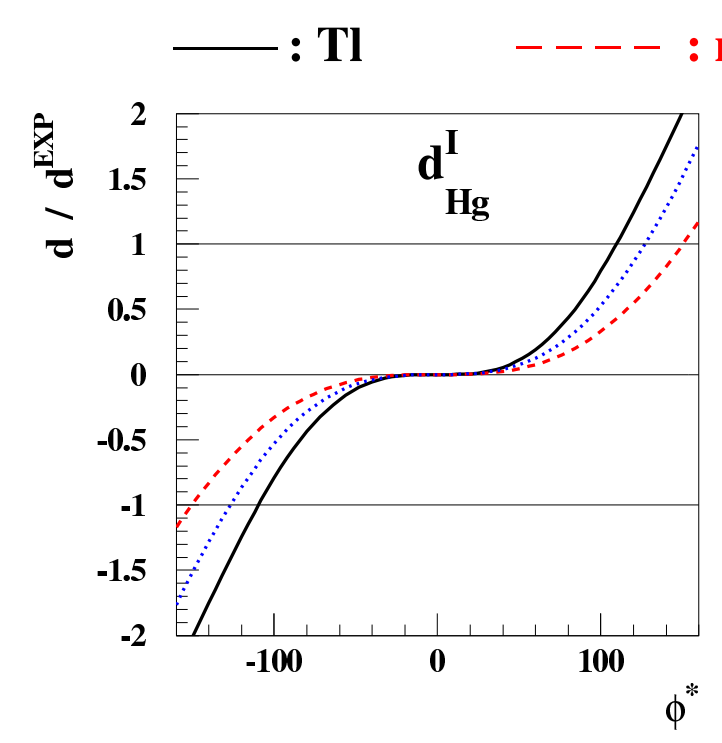

$\mathrm{n}(\mathbf{Q C D})$

: Hg
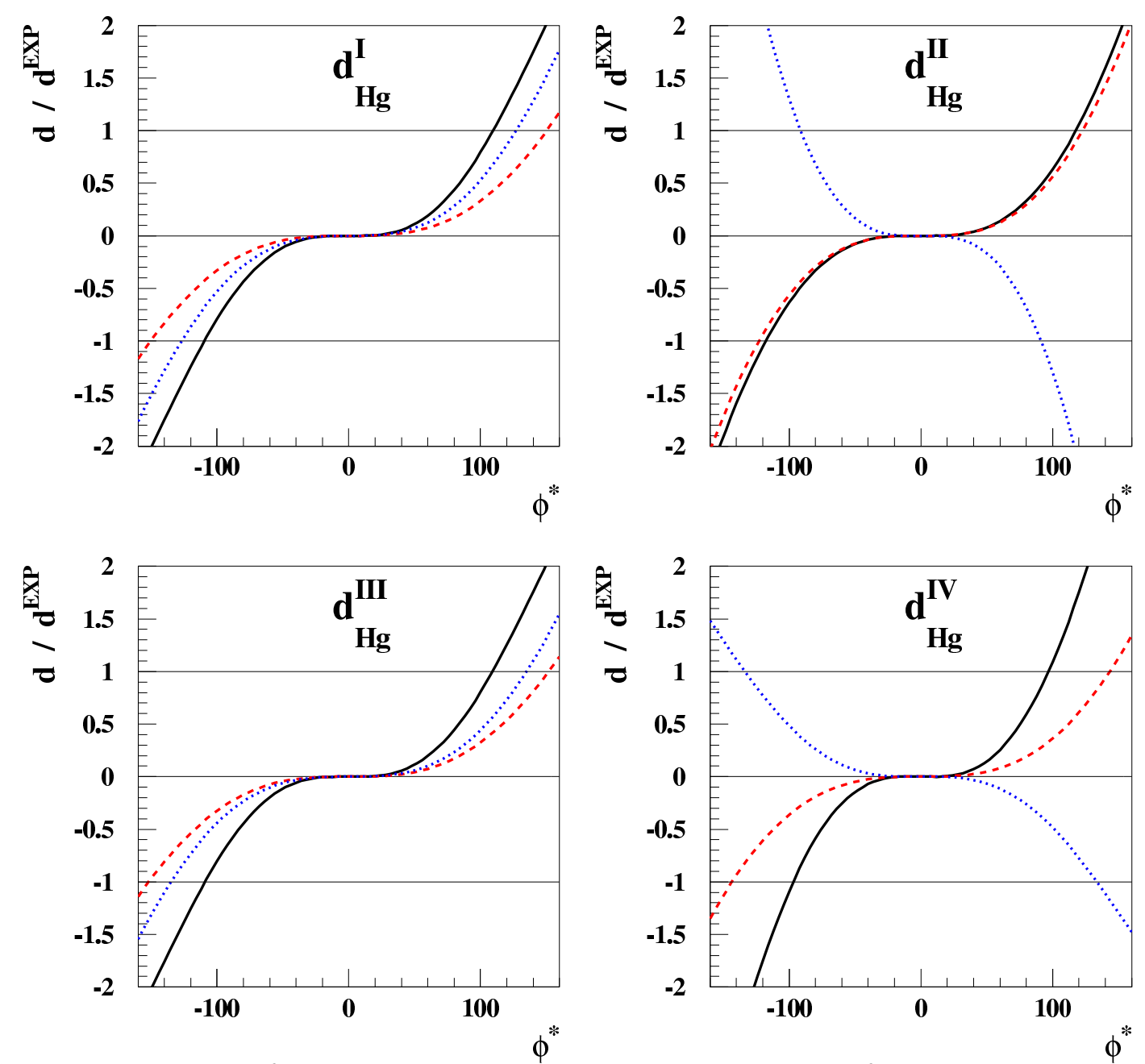

Figure 6. The values of the three EDMs along the directions optimized for $d_{\mathrm{Ra}}$, as obtained using $d_{\mathrm{Hg}}^{\mathrm{I}}$ (upper left), $d_{\mathrm{Hg}}^{\mathrm{II}}$ (upper right), $d_{\mathrm{Hg}}^{\mathrm{III}}$ (lower right), and $d_{\mathrm{Hg}}^{\mathrm{IV}}$ (lower left) for the Mercury EDM. The ratios $d_{\mathrm{Tl}} / d_{\mathrm{Tl}}^{\mathrm{EXP}}, d_{\mathrm{n}} / d_{\mathrm{n}}^{\mathrm{EXP}}$, and $d_{\mathrm{Hg}} / d_{\mathrm{Hg}}^{\mathrm{EXP}}$ are denoted by the black solid, red dashed, and blue dotted lines, respectively. The scenario (4.1) is assumed, with the choice $\tan \beta=40$.

two reference directions with $\Phi_{1, A_{e}}=0$ and $\Phi_{2,3}=0$. We note in particular that, in the case of $d_{\mathrm{Hg}}^{\mathrm{IV}}$, the Radium EDM may become about an order of magnitude larger than is possible with another choices for the Mercury EDM calculation.

Figure 6 shows the Thallium (solid), neutron (dashed) and Mercury (dotted) EDMs along the directions chosen to optimize $d_{\mathrm{Ra}}$ for the four choices of $d_{\mathrm{Hg}}$ when $\tan \beta=40$. We see that $\phi^{*}$ could be as large as about $100^{\circ}$, with the most important constraints being provided by the Thallium (Mercury) EDM in the cases where the $d_{\mathrm{Hg}}^{\mathrm{I}, \mathrm{III}, \mathrm{IV}}\left(d_{\mathrm{Hg}}^{\mathrm{II}}\right)$ are used, respectively.

In figure 7 , we show the maximal values of $d_{\mathrm{Ra}}$ attainable in the $6 \mathrm{D}$ case after imposing the three EDM constraints. We see the that small-phase approximations for $d_{\mathrm{Hg}}^{\mathrm{I}, \mathrm{II}, \mathrm{III}}$ break down for $\phi^{*} \sim 40^{\circ}$, as seen by comparing with the calculations along the two reference 


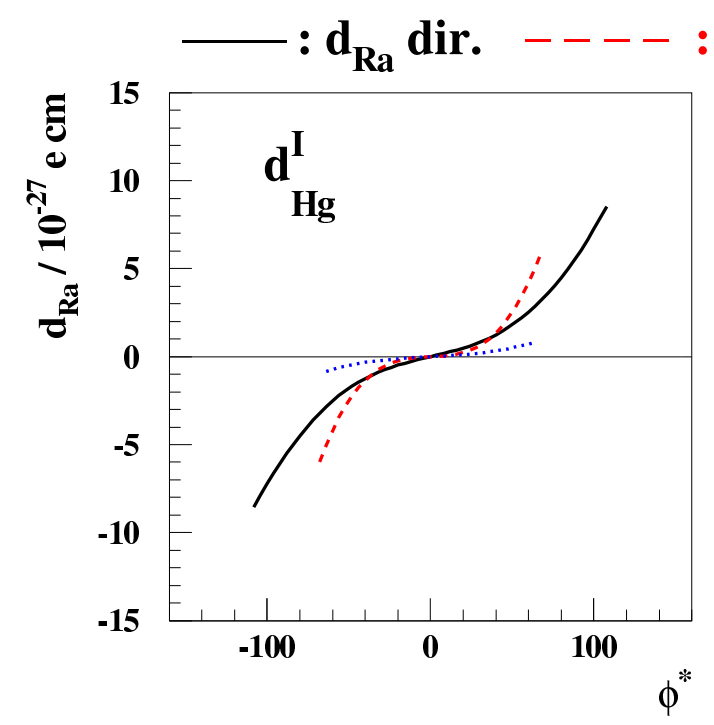

$\Phi_{1, \mathrm{Ae}}=\mathbf{0}$ dir. : $\Phi_{2,3}=\mathbf{0}$ dir.
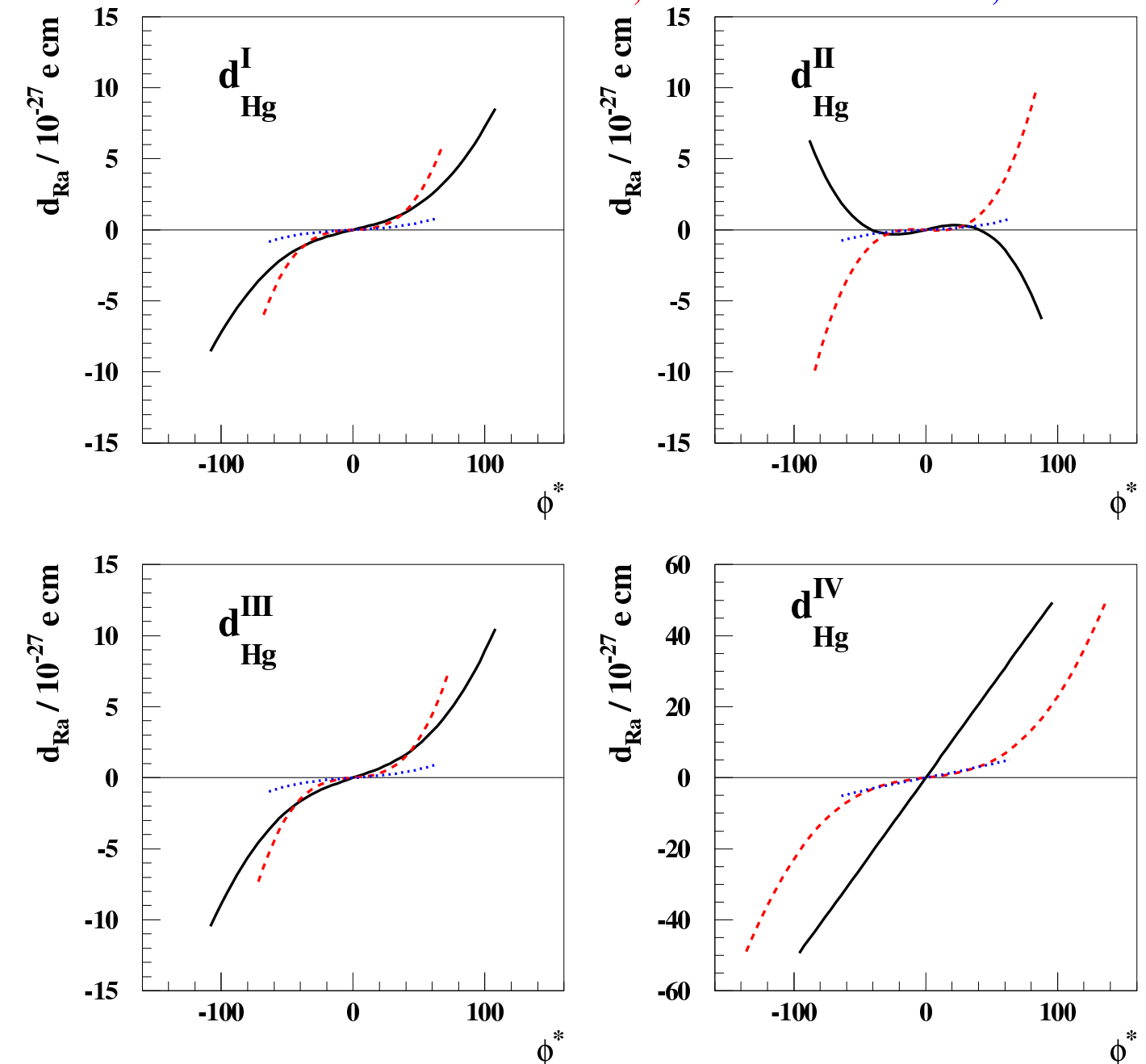

Figure 7. The EDM of ${ }^{225} \mathrm{Ra}$ in units of $10^{-27} e \mathrm{~cm}$ for the scenario (4.1) with $\tan \beta=40$ along the directions optimized for $d_{\mathrm{Ra}}$ (black solid) taking $d_{\mathrm{Hg}}^{\mathrm{I}}$ (upper left), $d_{\mathrm{Hg}}^{\mathrm{II}}$ (upper right), $d_{\mathrm{Hg}}^{\mathrm{III}}$ (lower right), and $d_{\mathrm{Hg}}^{\mathrm{IV}}$ (lower left) for the Mercury EDM. We have imposed the EDM constraints $\left|d_{\mathrm{Tl}} / d_{\mathrm{Tl}}^{\mathrm{EXP}}\right| \leq 1,\left|d_{\mathrm{n}} / d_{\mathrm{n}}^{\mathrm{EXP}}\right| \leq 1$, and $\left|d_{\mathrm{Hg}} / d_{\mathrm{Hg}}^{\mathrm{EXP}}\right| \leq 1$. For comparison, we also show $d_{\mathrm{Ra}}$ along two reference directions with the choices $\Phi_{1, A_{e}}=0$ (red dashed) and $\Phi_{2,3}=0$ (blue dotted).

directions, and that $d_{\mathrm{Ra}}$ can be as large as $\sim 6 \times 10^{-27} e \cdot \mathrm{cm}$ or more. On the other hand, in the case of $d_{\mathrm{Hg}}^{\mathrm{IV}}, d_{\mathrm{Ra}}$ can be as large as $\sim 50 \times 10^{-27} e \cdot \mathrm{cm}$.

Finally, in figure 8 we show the $6 \mathrm{CP}$-violating phases at the GUT scale (top and middle) and the $3 \mathrm{CP}$-violating phases of the third-generation $A$ parameters at the SUSY scale (bottom). We observe that the CP phases of the gaugino mass parameters $\Phi_{1}, \Phi_{2}$ and $\Phi_{3}$ can only be as large as $2^{\circ}, 0.4^{\circ}$ and $0.4^{\circ}$, respectively, whereas $\Phi_{A_{u}}\left(\Phi_{A_{d}}\right)$ at the GUT scale can be as large as $\sim 100^{\circ}\left(60^{\circ}\right)$, as previously seen in figure 4 . These CP-violating phases are suppressed at the SUSY scale by RG running from the GUT scale [3], but sizeable non-trivial CP-violating phases are still allowed at the SUSY scale: $\left|\Phi_{A_{t}}-180^{\circ}\right| \sim 2^{\circ},\left|\Phi_{A_{b}}-180^{\circ}\right| \sim 4^{\circ}$, and $\left|\Phi_{A_{\tau}}-180^{\circ}\right| \sim 30^{\circ}$. 


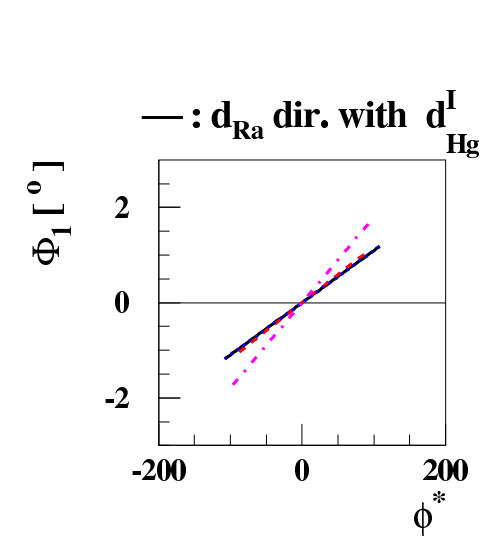

$-\cdot-: d_{\mathrm{Ra}}$ dir. with $\mathrm{d}_{\mathrm{Hg}}^{\mathrm{IV}}$
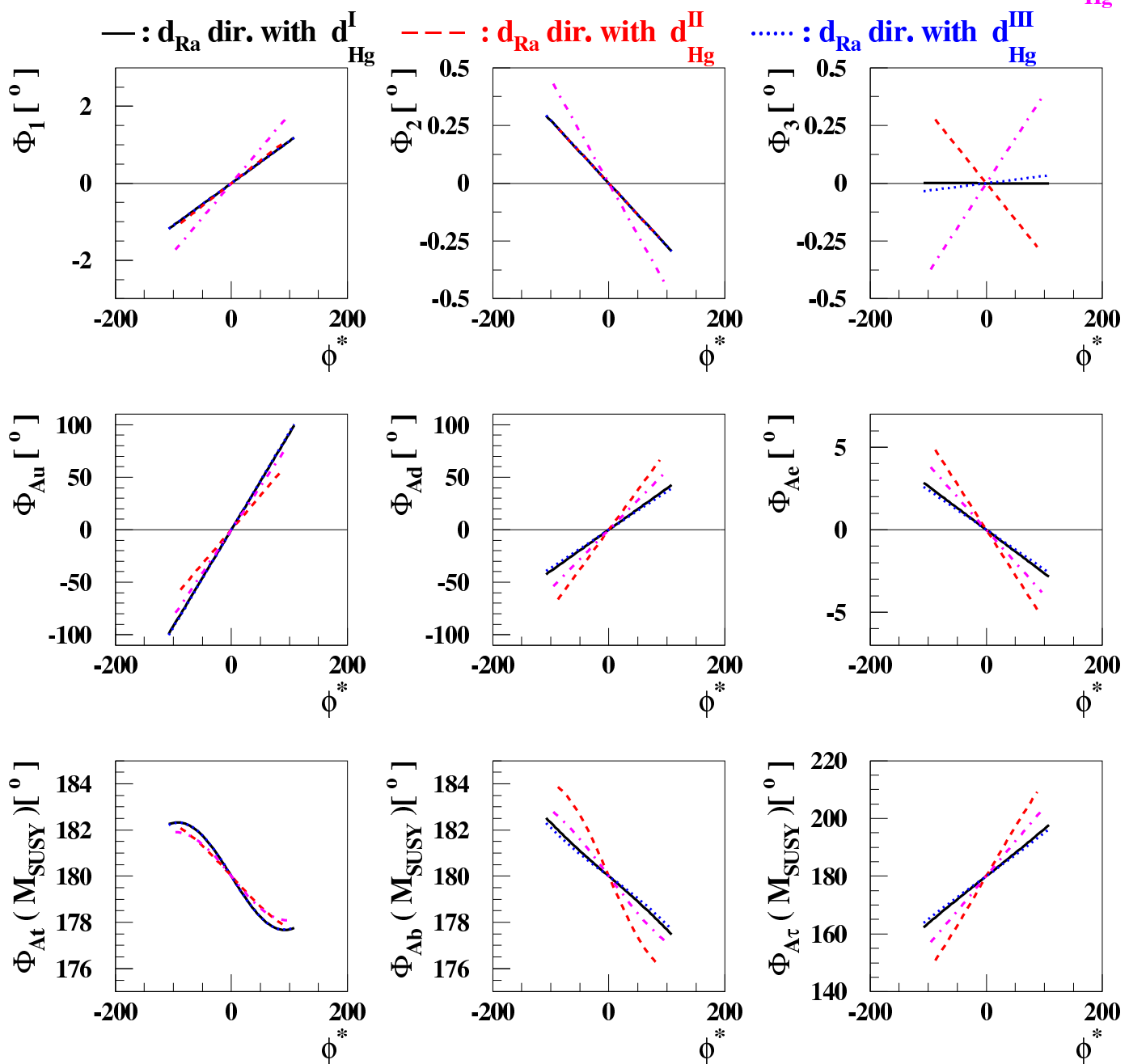

Figure 8. The $6 \mathrm{CP}$-violating phases at the GUT scale (upper and middle) and the $3 \mathrm{CP}$-violating phases of the third-generation $A$ parameters at the SUSY scale (lower) along the directions optimized for $d_{\mathrm{Ra}}$, as obtained using $d_{\mathrm{Hg}}^{\mathrm{I}}$ (solid black), $d_{\mathrm{Hg}}^{\mathrm{II}}$ (red dashed), $d_{\mathrm{Hg}}^{\mathrm{III}}$ (blue dotted), and $d_{\mathrm{Hg}}^{\mathrm{IV}}$ (magenta dash-dootted) for the Mercury EDM. We have imposed the EDM constraints $\left|d_{\mathrm{Tl}} / d_{\mathrm{Tl}}^{\mathrm{EX}}\right| \leq 1$, $\left|d_{\mathrm{n}} / d_{\mathrm{n}}^{\mathrm{EXP}}\right| \leq 1$, and $\left|d_{\mathrm{Hg}} / d_{\mathrm{Hg}}^{\mathrm{EXP}}\right| \leq 1$. The scenario (4.1) is assumed, with the choice $\tan \beta=40$.

\subsection{The $7 \mathrm{D}$ case of non-zero $\theta_{\mathrm{QCD}}$}

Hitherto, we have implicitly assumed that the CP-violating QCD $\theta$-term:

$$
\mathcal{L}=\frac{\alpha_{s}}{8 \pi} \bar{\theta} G_{\mu \nu}^{a} \tilde{G}^{\mu \nu, a}
$$

is negligible, where $\tilde{G}^{\mu \nu, a} \equiv \epsilon^{\mu \nu \rho \sigma} G_{\rho \sigma}^{a} / 2$ and the parameter $\bar{\theta}$ is given by the sum of the $\mathrm{QCD} \theta_{\mathrm{QCD}}$ and the strong chiral phase for the quark mass matrix:

$$
\bar{\theta}=\theta_{\mathrm{QCD}}+\operatorname{Arg} \operatorname{Det} M_{q} .
$$

In the weak basis where $\operatorname{Arg} \operatorname{Det} M_{q}=0$, we have $\bar{\theta}=\theta_{\mathrm{QCD}}$. 
The dimension-four operator (4.8) would in general contribute to the neutron, Mercury and Radium EDMs, e.g., through the CP-odd pion-nucleon-nucleon interactions (2.2). Explicitly, for the neutron EDM, we use the estimate $[12,18]$

$$
d_{\mathrm{n}}(\bar{\theta}) \simeq 2.5 \times 10^{-16} \bar{\theta} e \cdot \mathrm{cm}
$$

For the $\bar{\theta}$-induced Mercury EDM, we neglect the contribution from the $\bar{g}_{\pi N N}^{(0)}$ coupling and use

$$
d_{\mathrm{Hg}}^{\mathrm{I}, \mathrm{II}, \mathrm{III}, \mathrm{IV}}(\bar{\theta}) \simeq\left(C_{\mathrm{Hg}}^{\mathrm{I}, \mathrm{II}, \mathrm{III}, \mathrm{IV}} \times 10^{-3} \mathrm{GeV}^{-1}\right) e \bar{g}_{\pi N N}^{(1)}(\bar{\theta}),
$$

where $C_{\mathrm{Hg}}^{\mathrm{I}}=1.8, C_{\mathrm{Hg}}^{\mathrm{II}}=1.0, C_{\mathrm{Hg}}^{\mathrm{III}}=1.4$ and $C_{\mathrm{Hg}}^{\mathrm{IV}}=9.5 \times 10^{-2}$, with $[12,45]$

$$
\bar{g}_{\pi N N}^{(1)}(\bar{\theta}) \simeq 1.1 \times 10^{-3} \bar{\theta}
$$

Finally, for the Radium EDM, we use

$$
d_{\mathrm{Ra}}(\bar{\theta}) \simeq\left(3.5 \times 10^{-1} \mathrm{GeV}^{-1}\right) e \bar{g}_{\pi N N}^{(1)}(\bar{\theta}) .
$$

Henceforward, we normalize $\bar{\theta}$ in units of $10^{-10}$ :

$$
\widehat{\theta} \equiv \bar{\theta} \times 10^{10} .
$$

With this normalization, when $\widehat{\theta}=1$, we have $d_{\mathrm{n}}(\bar{\theta})=2.5 \times 10^{-26} \mathrm{e} \cdot \mathrm{cm}$, which is very close to the current experimental bound $d_{\mathrm{n}}^{\mathrm{EXP}}=3 \times 10^{-26} e \cdot \mathrm{cm}$, and we find $d_{\mathrm{Ra}} \simeq$ $0.8 \times 10^{-27} e \cdot \mathrm{cm}$.

We have analyzed the possible maximal values of $d_{\mathrm{Ra}}$ in this 7D case including $\widehat{\theta}$ following a procedure similar to that we used in the $6 \mathrm{D}$ case. Looking again at figure 1 , we note the horizontal magenta lines representing the 7th components of the vectors representing the present EDM constraints on $d_{\mathrm{Tl}}$ (upper left), $d_{\mathrm{n}}$ (upper right), and $d_{\mathrm{Hg}}$ (lower left), and of the vector representing the EDM of ${ }^{225} \mathrm{Ra}$ (lower right). We observe that the 7th component is missing in the $d_{\mathrm{Tl}}$ case, because this observable has no contribution from the $\widehat{\theta}$ term in our approach. On the other hand, the $\widehat{\theta}$ component is close to unity for $d_{\mathrm{n}}$ (cf. the discussion at the end of the previous paragraph), $\sim 10^{-1}$ for $d_{\mathrm{Hg}}^{\mathrm{I}}$, and somewhat less than unity for $d_{\text {Ra }}$. This implies that a measurement of $d_{\text {Ra }}$ at the level of $10^{-27} e \mathrm{~cm}$ would already be a competitive measurement of $\widehat{\theta}$, even in the absence of the other MCPMFV phases.

As seen in figure 2, we have made similar analyses using the $d_{\mathrm{Hg}}^{\mathrm{II}, \mathrm{III} \text {,IV }}$ calculations, finding similar $\widehat{\theta}$ components in the first two cases, but a value about an order of magnitude smaller in the $d_{\mathrm{Hg}}^{\mathrm{IV}}$ case. We have also analyzed (not shown) the cosines of the angles between the observable vector $\mathbf{O}^{d_{\mathrm{Ra}}}$ and the EDM-constraint vectors $\mathbf{E}^{d_{\mathrm{Tl}}}, \mathbf{E}^{d_{\mathrm{n}}}$ and $\mathbf{E}^{\mathrm{d}_{\mathrm{Hg}}^{\mathrm{I}, \mathrm{II}, \mathrm{III}, \mathrm{IV}}}$ in the 7D model, as functions of $\tan \beta$. We find results that are very similar to the $6 \mathrm{D}$ case shown in figure 3 , the most significant difference being quite small and limited to $\tan \beta<10$ in the $d_{\mathrm{n}}$ case.

We turn now to figure 9, which is the analogue of figure 4, but including the QCD $\theta$ term (4.8). We see significant differences at intermediate and large $\tan \beta$, where we 

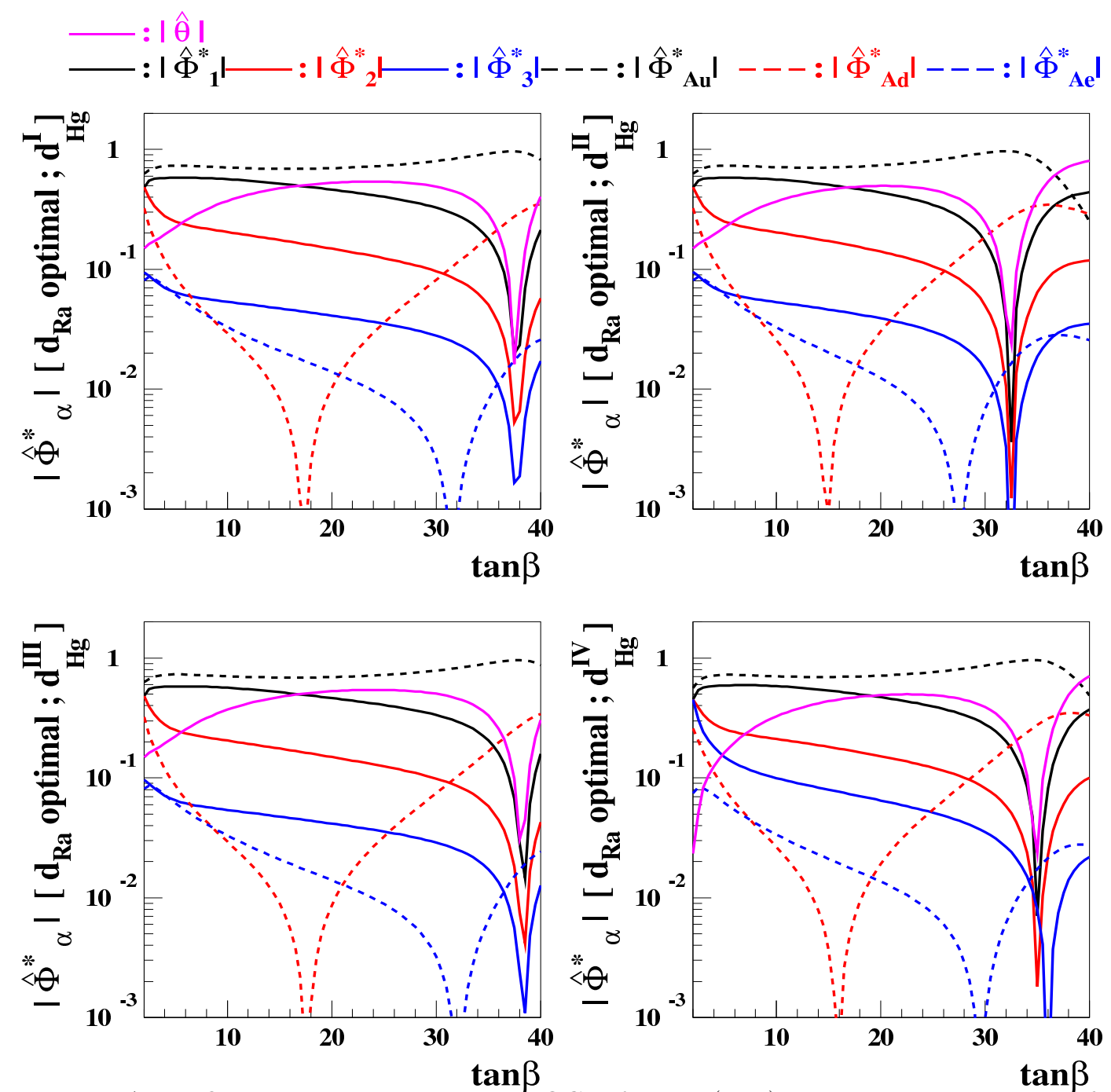

Figure 9. As in figure 4, but including the QCD $\theta$ term (4.8). The 7 th components of the normalized 7D optimal vectors are denoted by the solid magenta lines.

note that the optimal vectors in all four models for $d_{\mathrm{Hg}}$ exhibit not only relatively large $\widehat{\theta}$ components, but also larger components for the gaugino mass phases than in the $6 \mathrm{D}$ case. We have also analyzed (not shown) the products $\widehat{\Phi}^{*} \cdot \mathbf{O}$ for the optimal $d_{\mathrm{Ra}}$ direction in the $7 \mathrm{D}$ space, as obtained using the calculations $d_{\mathrm{Hg}}^{\mathrm{I}, \mathrm{II}, \mathrm{III}, \mathrm{IV}}$ for the Mercury EDM, finding results that are generally very similar to those shown in figure 5 with a little rise around $\tan \beta=40$.

Figure 10 displays the values of the Thallium, neutron and Mercury EDMs along the directions optimized for $d_{\text {Ra }}$, analogously to figure 6 but this time in 7D. As before, the different panels display results obtained using $d_{\mathrm{Hg}}^{\mathrm{I}}$ (upper left), $d_{\mathrm{Hg}}^{\mathrm{II}}$ (upper right), $d_{\mathrm{Hg}}^{\mathrm{III}}$ (lower right), and $d_{\mathrm{Hg}}^{\mathrm{IV}}$ (lower left) for the Mercury EDM, and the ratios $d_{\mathrm{Tl}} / d_{\mathrm{Tl}}^{\mathrm{EXP}}, d_{\mathrm{n}} / d_{\mathrm{n}}^{\mathrm{EXP}}$, and $d_{\mathrm{Hg}} / d_{\mathrm{Hg}}^{\mathrm{EXP}}$ are denoted by the black solid, red dashed, and blue dotted lines, respectively. Also as before, the scenario (4.1) is assumed, with the choice $\tan \beta=40$. Comparing with figure 6, we see that the magnitudes of the Thallium and Mercury EDMs increase quickly as $\phi^{*}$ deviates from 0 and it could be as large as only about $50^{\circ}$. 


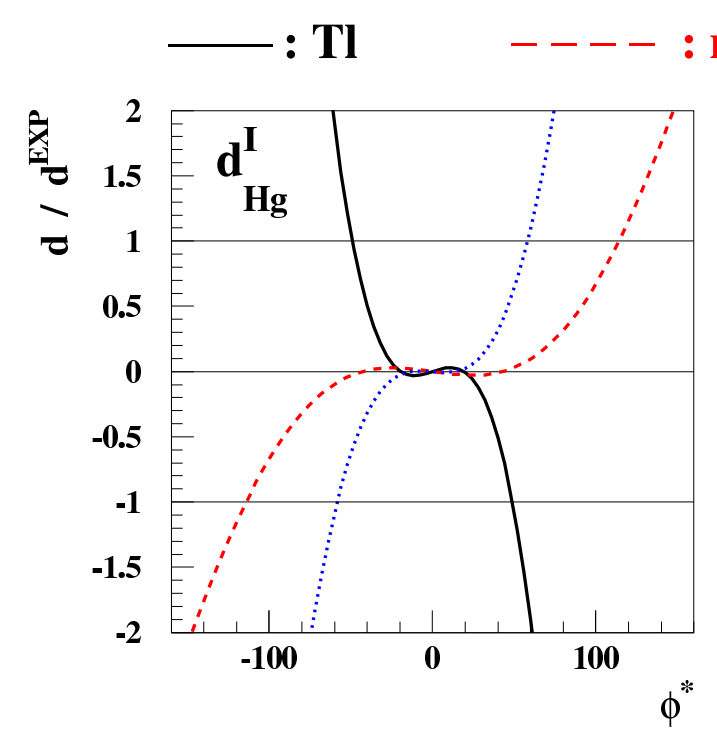

$\mathrm{n}(\mathbf{Q C D})$
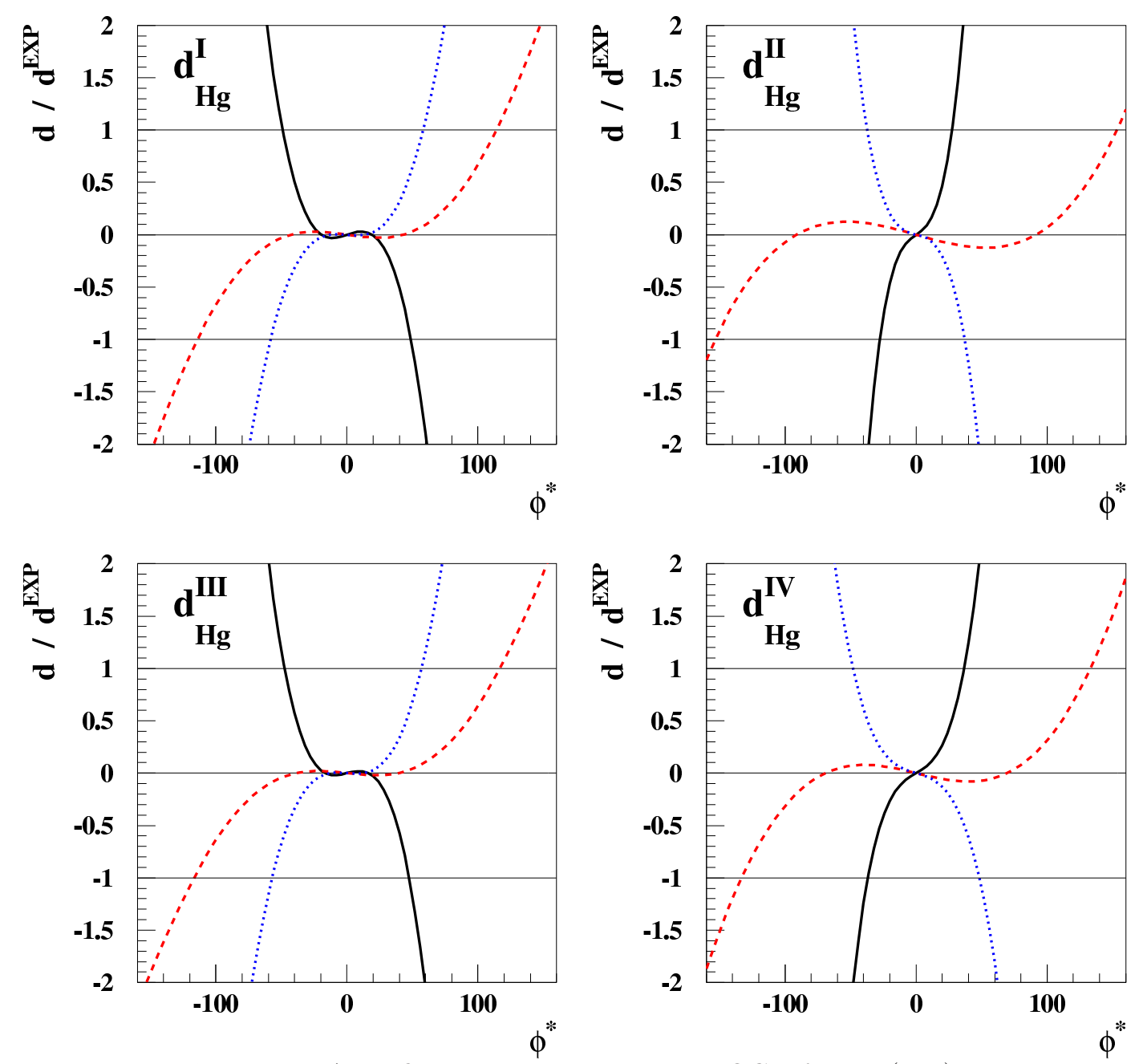

Figure 10. As in figure 6, but including the QCD $\theta$ term (4.8).

Turning to the resulting 7D estimates of $d_{\mathrm{Ra}}$ shown in figure 11, we see that they have somewhat smaller magnitudes than the $6 \mathrm{D}$ estimates shown in figure 7 in the linear approximation. However, larger values of the $\mathrm{CP}$-violating gaugino phases are allowed in the $7 \mathrm{D}$ case than in the $6 \mathrm{D}$ case, as seen by comparing figures 8 and 12 . Specifically, we observe that $\Phi_{1}, \Phi_{2}$ and $\Phi_{3}$ could be as large as $\sim 15^{\circ}, \sim 4^{\circ}$ and $\sim 1^{\circ}$, respectively. We also see in figure 13 that sizeable $\bar{\theta}$ could be much larger than the upper limit of $\sim 10^{-10}$ usually quoted, with values as large as $\bar{\theta} \sim 2.5 \times 10^{-9}$ becoming possible in the presence of non-zero MCPMFV phases.

\section{Conclusions}

In this paper we have extended our previous analyses of the MCPMFV model, with its 6 CP-violating phases to determine (in the linear approximation) the largest value of $d_{\mathrm{Ra}}$ that is allowed by the present constraints on the neutron, Thallium and Mercury EDMs, using 

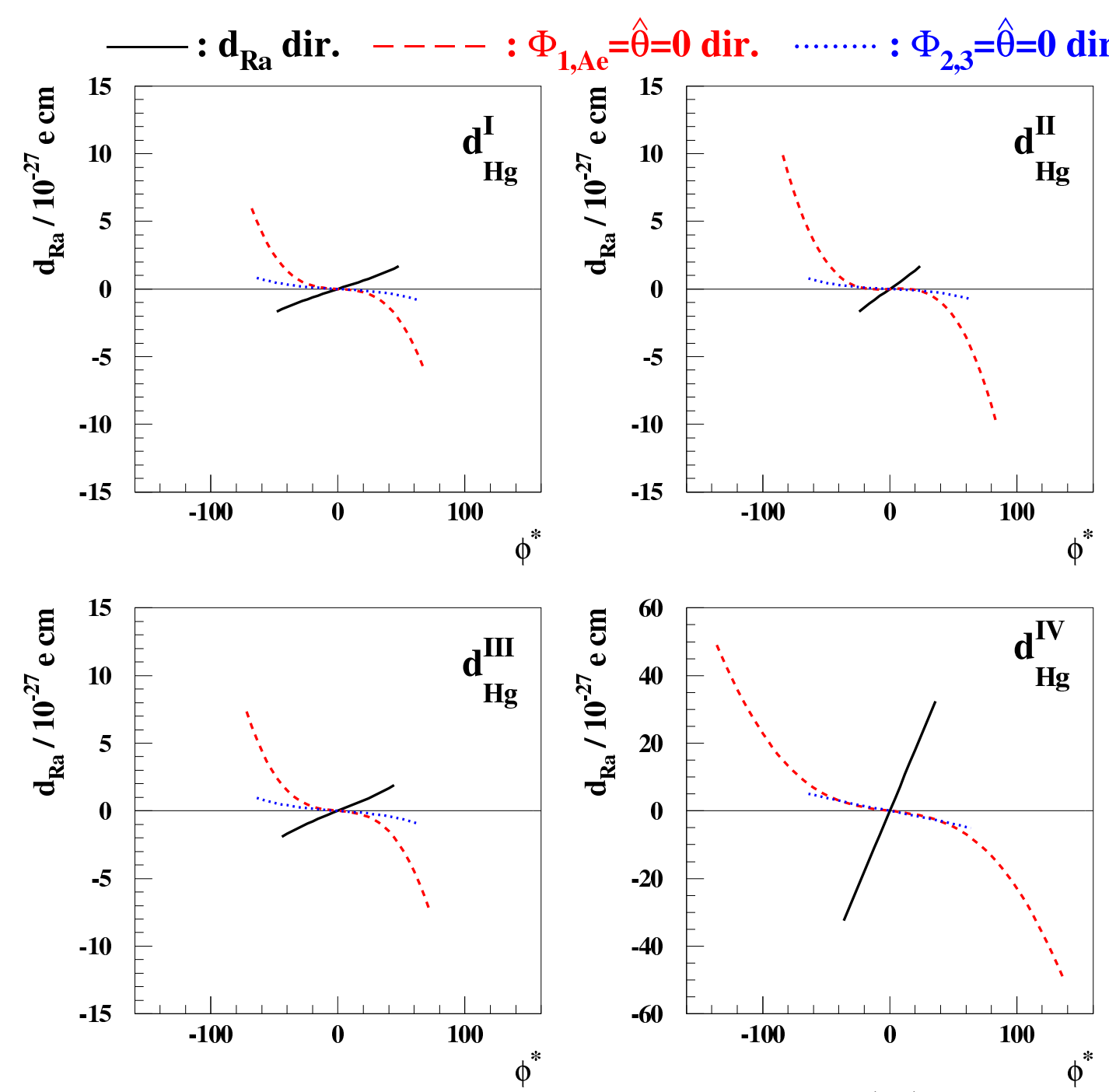

Figure 11. As in figure 7, but including the QCD $\theta$ term (4.8).

the differential-geometric approach developed in [12]. Numerically, we obtain rather similar results whether we include the $\mathrm{CP}$-violating $\mathrm{QCD}$ vacuum phase $\bar{\theta}$ in the analysis, or not.

The results are much more sensitive to the theoretical treatment of the Mercury EDM constraint, and we compare the results obtained with four different calculations of $d_{\mathrm{Hg}}$. Three of them yield quite similar results for $d_{\mathrm{Ra}}$, but one calculations indicates a smaller dependence of $d_{\mathrm{Hg}}$ on the CP-violating phases of the MCPMFV model, and hence allows larger numerical values of these phase and, in general, larger values of $d_{\text {Ra }}$ become possible. The maximal values we find for $d_{\mathrm{Ra}}$ using three of the $d_{\mathrm{Hg}}$ calculations are typically $\sim$ $6 \times 10^{-27} \mathrm{e} \cdot \mathrm{cm}$ or more, whereas the fourth calculation allows $d_{\mathrm{Ra}} \sim 50 \times 10^{-27} \mathrm{e} \cdot \mathrm{cm}$.

For comparison, we recall that there is a proposal to measure $d_{\text {Ra }}$ with an sensitivity approaching $\sim 10^{-27} e \cdot \mathrm{cm}$ in one day of data-taking. This experiment would clearly have interesting potential to probe regions of the MCPMFV parameter space that have not been explored by previous EDM experiments.

This potential surely extends to many other models with several sources of CP violation, which could also be analyzed using the differential-geometric approach [12] exploited 

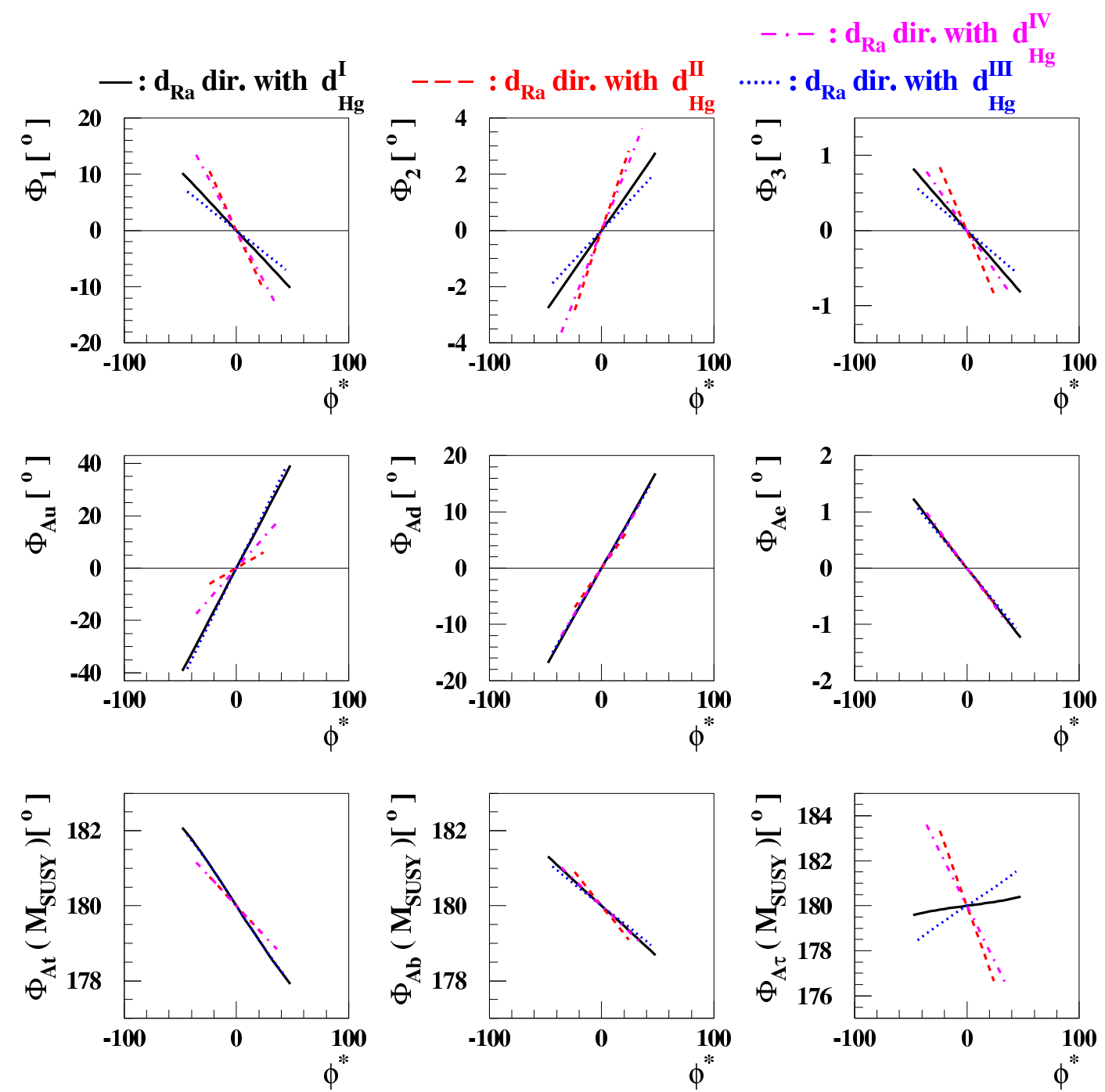

Figure 12. As in figure 8, but including the QCD $\theta$ term (4.8).

here. As long as a limited number $n$ of EDMs have been bounded (or measured) by experiments, any model with $N>n$ CP-violating parameters will be underconstrained, and (partial) cancellations $[10,11]$ are possible that would allow large values for other CPviolating observables. The MCPMFV model is one such example, in which $N=6$ (or 7 if $\bar{\theta}$ is included) and $n=3$, so far. As we have shown in this paper using this differentialgeometric approach, constraining or measuring $d_{\mathrm{Ra}}$ at the level of $\sim 10^{-27} \mathrm{e} \cdot \mathrm{cm}$ or better would be a valuable addition to the existing arsenal of experimental probes of $\mathrm{CP}$ violation.

However, it would still not complete the set of constraints needed for a model with $N>4$, such as the MCPMFV. For this reason, other measurements, e.g., of the CPviolating asymmetry in $b \rightarrow s \gamma$ decay. We have discussed elsewhere the maximal value that this observable might take in the MCPMFV, and it would be an interesting and complementary measurement to that of $d_{\text {Ra }}$.

We conclude by drawing reader's attention to potential caveats in the physics of CP violation. The baryon asymmetry of the Universe is one of the strongest pieces of evidence 


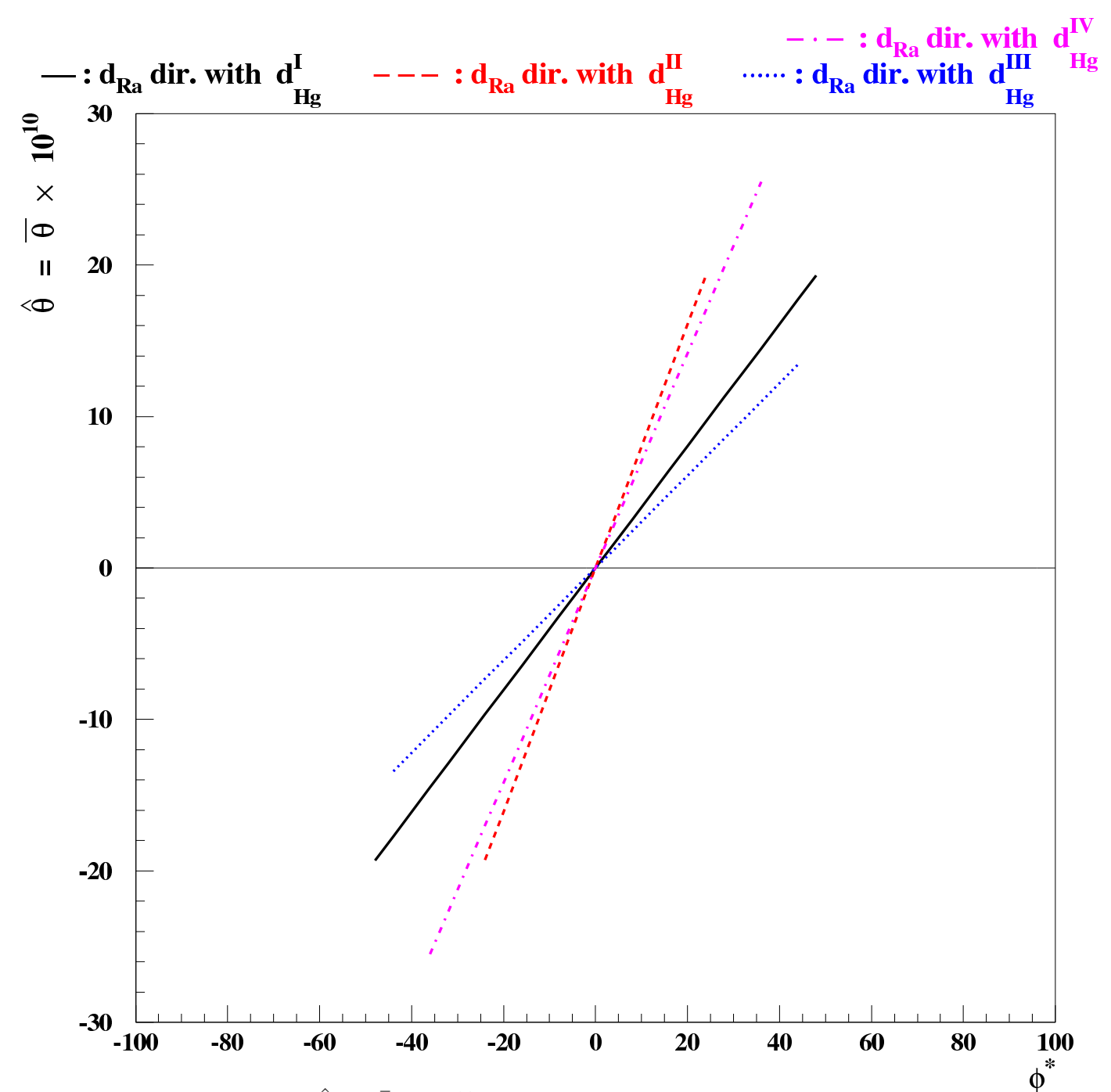

Figure 13. The values of $\hat{\theta} \equiv \bar{\theta} \times 10^{10}$ along the directions that maximizer $d_{\mathrm{Ra}}$ in the linear approximation, as found by using the calculations $d_{\mathrm{Hg}}^{\mathrm{I}}$ (solid black), $d_{\mathrm{Hg}}^{\mathrm{II}}$ (red dashed), $d_{\mathrm{Hg}}^{\mathrm{III}}$ (blue dotted), and $d_{\mathrm{Hg}}^{\mathrm{IV}}$ (magenta dash-dootted) for the Mercury EDM. We have imposed the EDM constraints $\left|d_{\mathrm{Tl}} / d_{\mathrm{Tl}}^{\mathrm{EXP}}\right| \leq 1,\left|d_{\mathrm{n}} / d_{\mathrm{n}}^{\mathrm{EXP}}\right| \leq 1$, and $\left|d_{\mathrm{Hg}} / d_{\mathrm{Hg}}^{\mathrm{EXP}}\right| \leq 1$. The scenario (4.1) is assumed, fixing $\tan \beta=40$.

for physics beyond the Standard Model, as it cannot be generated successfully within the standard Kobayashi-Maskawa model of $\mathrm{CP}$ violation. There must be new sources of $\mathrm{CP}$ violation beyond the Kobayashi-Maskawa phase, and it behooves experiments to chase down all those within reach. Some CP-violating phases may manifest themselves at the TeV scale and be accessible to contemporary collider experiments, e.g. at the LHC. However, baryogenesis could equally well be achieved via CP-violating phases appearing at higher energy scales, and EDMs have the potential to probe beyond the $\mathrm{TeV}$ scale, in particular because the Standard Model Kobayashi-Maskawa predictions for EDMs are quite small. As our analysis exemplifies, new EDM observables probe complementary region of parameter space including the strong $\mathrm{CP}$ phase $\theta_{\mathrm{QCD}}$. Therefore, constraining or even measuring $d_{\mathrm{Ra}}$ is an interesting experimental objective. 


\section{Acknowledgments}

We thank Jon Engel, Maxim Pospelov and Adam Ritz for useful comments and suggestions.

Open Access. This article is distributed under the terms of the Creative Commons Attribution Noncommercial License which permits any noncommercial use, distribution, and reproduction in any medium, provided the original author(s) and source are credited.

\section{References}

[1] T. Ibrahim and P. Nath, CP violation from standard model to strings, Rev. Mod. Phys. 80 (2008) 577 [arXiv: 0705. 2008] [SPIRES].

[2] P. Nath et al., The hunt for new physics at the Large Hadron Collider, Nucl. Phys. Proc. Suppl. 200-202 (2010) 185 [arXiv:1001.2693] [SPIRES].

[3] J.R. Ellis, J.S. Lee and A. Pilaftsis, B-meson observables in the maximally CP-violating MSSM with minimal flavour violation, Phys. Rev. D 76 (2007) 115011 [arXiv:0708.2079] [SPIRES].

[4] M. Argyrou, A.B. Lahanas and V.C. Spanos, Refining the predictions of supersymmetric CP-violating models: a top-down approach, JHEP 05 (2008) 026 [arXiv:0804.2613] [SPIRES].

[5] G. Colangelo, E. Nikolidakis and C. Smith, Supersymmetric models with minimal flavour violation and their running, Eur. Phys. J. C 59 (2009) 75 [arXiv:0807.0801] [SPIRES].

[6] W. Altmannshofer, A.J. Buras and P. Paradisi, Low energy probes of CP-violation in a flavor blind MSSM, Phys. Lett. B 669 (2008) 239 [arXiv:0808.0707] [SPIRES].

[7] L. Mercolli and C. Smith, EDM constraints on flavored CP-violating phases, Nucl. Phys. B 817 (2009) 1 [arXiv:0902.1949] [SPIRES].

[8] A.L. Kagan, G. Perez, T. Volansky and J. Zupan, General minimal flavor violation, Phys. Rev. D 80 (2009) 076002 [arXiv: 0903. 1794] [SPIRES].

[9] R. Zwicky and T. Fischbacher, On discrete minimal flavour violation, Phys. Rev. D 80 (2009) 076009 [arXiv:0908.4182] [SPIRES].

[10] T. Ibrahim and P. Nath, The neutron and the lepton EDMs in MSSM, large CP-violating phases and the cancellation mechanism, Phys. Rev. D 58 (1998) 111301 [Erratum ibid. D 60 (19999) 099902] [hep-ph/9807501] [SPIRES].

[11] M. Brhlik, L.L. Everett, G.L. Kane and J.D. Lykken, A resolution to the supersymmetric CP problem with large soft phases via D-branes, Phys. Rev. Lett. 83 (1999) 2124 [hep-ph/9905215] [SPIRES].

[12] J. Ellis, J.S. Lee and A. Pilaftsis, A geometric approach to CP-violation: applications to the MCPMFV SUSY model, JHEP 10 (2010) 049 [arXiv: 1006.3087] [SPIRES].

[13] J. Ellis, J.S. Lee and A. Pilaftsis, Note on a differential-geometrical construction of optimal directions in linearly-constrained systems, arXiv:1009.1151 [SPIRES].

[14] L.I. Schiff, Measurability of nuclear electric dipole moments, Phys. Rev. 132 (1963) 2194.

[15] L. Willmann, K. Jungmann and H.W. Wilschut, Searches for permanent electric dipole moments in Radium Isotopes, Letter of Intent to the ISOLDE and Neutron Time-of-Flight Experiments Committee for experiments with HIE-ISOLDE, CERN-INTC-2010-049/INTC-I-115. 
[16] J. Pakarinen et al., Measurements of octupole collectivity in odd-mass Rn, Fr and Ra isotopes, Letter of Intent to the ISOLDE and Neutron Time-of-Flight Experiments Committee for experiments with HIE-ISOLDE, CERN-INTC-2010-022/INTC-I-091.

[17] I.B. Khriplovich and R.A. Korkin, $P$ and $T$ odd electromagnetic moments of deuteron in chiral limit, Nucl. Phys. A 665 (2000) 365 [nucl-th/9904081] [SPIRES].

[18] M. Pospelov and A. Ritz, Electric dipole moments as probes of new physics, Annals Phys. 318 (2005) 119 [hep-ph/0504231] [SPIRES].

[19] S. Ban, J. Dobaczewski, J. Engel and A. Shukla, Fully self-consistent calculations of nuclear Schiff moments, Phys. Rev. C 82 (2010) 015501 [arXiv: 1003. 2598] [SPIRES].

[20] V.F. Dmitriev and R.A. Sen'kov, P- and T-violating Schiff moment of the Mercury nucleus, Phys. Atom. Nucl. 66 (2003) 1940 [Yad. Fiz. 66 (2003) 1988] [nucl-th/0304048] [SPIRES].

[21] J.R. Ellis, J.S. Lee and A. Pilaftsis, Electric dipole moments in the MSSM reloaded, JHEP 10 (2008) 049 [arXiv: 0808.1819] [SPIRES].

[22] J.S. Lee et al., CPsuperH: a computational tool for Higgs phenomenology in the minimal supersymmetric standard model with explicit CP-violation, Comput. Phys. Commun. 156 (2004) 283 [hep-ph/0307377] [SPIRES].

[23] J.S. Lee, M. Carena, J. Ellis, A. Pilaftsis and C.E.M. Wagner, CPsuperH2.0: an improved computational tool for Higgs phenomenology in the MSSM with explicit CP-violation, Comput. Phys. Commun. 180 (2009) 312 [arXiv:0712.2360] [SPIRES].

[24] J.H. de Jesus and J. Engel, Time-reversal-violating Schiff moment of ${ }^{1} 99 \mathrm{Hg}$, Phys. Rev. C 72 (2005) 045503 [nucl-th/0507031] [SPIRES].

[25] J. Engel, M. Bender, J. Dobaczewski, J.H. De Jesus and P. Olbratowski, Time-reversal violating Schiff moment of ${ }^{2} 25$ Ra, Phys. Rev. C 68 (2003) 025501 [nucl-th/0304075] [SPIRES].

[26] J. Dobaczewski and J. Engel, Nuclear time-reversal violation and the Schiff moment of ${ }^{2} 25$ Ra, Phys. Rev. Lett. 94 (2005) 232502 [nucl-th/0503057] [SPIRES].

[27] V.F. Dmitriev, R.A. Sen'kov and N. Auerbach, Effects of core polarization on the nuclear Schiff moment, Phys. Rev. C 71 (2005) 035501 [nucl-th/0408065] [SPIRES].

[28] M. Pospelov, Best values for the CP-odd meson nucleon couplings from supersymmetry, Phys. Lett. B 530 (2002) 123 [hep-ph/0109044] [SPIRES].

[29] M. Pospelov and A. Ritz, private communication.

[30] O. Lebedev and M. Pospelov, Electric dipole moments in the limit of heavy superpartners, Phys. Rev. Lett. 89 (2002) 101801 [hep-ph/0204359] [SPIRES].

[31] D.A. Demir, O. Lebedev, K.A. Olive, M. Pospelov and A. Ritz, Electric dipole moments in the MSSM at large $\tan \beta$, Nucl. Phys. B 680 (2004) 339 [hep-ph/0311314] [SPIRES].

[32] M.V. Romalis, W.C. Griffith and E.N. Fortson, A new limit on the permanent electric dipole moment of Hg-199, Phys. Rev. Lett. 86 (2001) 2505 [hep-ex/0012001] [SPIRES].

[33] W.C. Griffith et al., Improved limit on the permanent electric dipole moment of Hg-199, Phys. Rev. Lett. 102 (2009) 101601 [SPIRES].

[34] V.V. Flambaum, I.B. Khriplovich and O.P. Sushkov, Limit on the constant of T nonconserving nucleon nucleon interaction, Phys. Lett. B 162 (1985) 213 [SPIRES].

[35] V.V. Flambaum, I.B. Khriplovich and O.P. Sushkov, On the $P$ and $T$ nonconserving nuclear moments, Nucl. Phys. A 449 (1986) 750 [SPIRES]. 
[36] V.A. Dzuba, V.V. Flambaum, J.S.M. Ginges and M.G. Kozlov, Electric dipole moments of $H g, X e, R n, R a, P u$ and TlF induced by the nuclear Schiff moment and limits on timereversal violating interactions, Phys. Rev. A 66 (2002) 012111 [hep-ph/0203202] [SPIRES].

[37] K.V.P. Latha, D. Angom, B.P. Das and D. Mukherjee, Probing CP-violation with the electric dipole moment of atomic mercury, Phys. Rev. Lett. 103 (2009) 083001 [arXiv:0902.4790] [SPIRES].

[38] CMS collaboration, V. Khachatryan et al., Search for supersymmetry in pp collisions at $7 \mathrm{TeV}$ in events with jets and missing transverse energy, arXiv:1101.1628 [SPIRES].

[39] M. Battaglia et al., Proposed post-LEP benchmarks for supersymmetry, Eur. Phys. J. C 22 (2001) 535 [hep-ph/0106204] [SPIRES].

[40] B.C. Allanach et al., The Snowmass points and slopes: benchmarks for SUSY searches, Eur. Phys. J. C 25 (2002) 113 [hep-ph/0202233] [SPIRES].

[41] N. Ghodbane and H.-U. Martyn, Compilation of SUSY particle spectra from Snowmass 2001 benchmark models, in the proceedings of the APS/DPF/DPB Summer Study on the Future of Particle Physics, June 30-July 21, Snowmass, U.S.A. (2001), hep-ph/0201233 [SPIRES].

[42] M. Battaglia et al., Updated post-WMAP benchmarks for supersymmetry, Eur. Phys. J. C 33 (2004) 273 [hep-ph/0306219] [SPIRES].

[43] B.C. Regan, E.D. Commins, C.J. Schmidt and D. DeMille, New limit on the electron electric dipole moment, Phys. Rev. Lett. 88 (2002) 071805 [SPIRES].

[44] C.A. Baker et al., An improved experimental limit on the electric dipole moment of the neutron, Phys. Rev. Lett. 97 (2006) 131801 [hep-ex/0602020] [SPIRES].

[45] O. Lebedev, K.A. Olive, M. Pospelov and A. Ritz, Probing CP-violation with the deuteron electric dipole moment, Phys. Rev. D 70 (2004) 016003 [hep-ph/0402023] [SPIRES]. 